\title{
EMPIRICAL DETERMINATION OF CONVECTION PARAMETERS IN WHITE DWARFS. I. WHOLE EARTH TELESCOPE OBSERVATIONS OF EC14012-1446*
}

\author{
J. L. Provencal ${ }^{1,2}$, M. H. Montgomery ${ }^{2,3}$, A. KanaAn ${ }^{4}$, S. E. Thompson ${ }^{1,2,5}$, J. Dalessio ${ }^{1,2}$, H. L. Shipman $^{1,2}$, \\ D. Childers ${ }^{2,6}$, J. C. Clemens ${ }^{7}$, R. Rosen $^{8}$, P. Henrique $^{4}$, A. Bischoff-Kim ${ }^{9}$, W. Strickland ${ }^{10}$, D. Chandler ${ }^{10}$, B. Walter ${ }^{10}$, \\ T. K. Watson ${ }^{11}$, B. Castanheira ${ }^{12}$, S. Wang ${ }^{3}$, G. Handler ${ }^{12}$, M. Wood ${ }^{13}$, S. Vennes ${ }^{13}$, P. Nemeth ${ }^{13}$, S. O. KePleR $^{14}$,

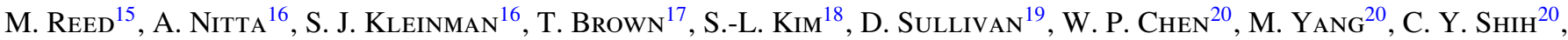 \\ X. J. JiAng ${ }^{21}$, A. V. Sergeev ${ }^{22}$, A. MaKsim ${ }^{22}$, R. Janulis ${ }^{23}$, K. S. BaliYan ${ }^{24}$, H. O. VAts ${ }^{24}$, S. Zola ${ }^{25,26}$, A. Baran ${ }^{25}$, \\ M. Winiarski ${ }^{25,26}$, W. Ogloza ${ }^{25,26}$, M. Paparo ${ }^{27}$, Z. BognaR ${ }^{27}$, P. Papics ${ }^{27}$, D. Kilkenny ${ }^{28}$, R. SEFAKo $^{29}$, D. Buckley $^{29}$,
} N. Loaring ${ }^{29}$, A. Kniazev ${ }^{29}$, R. Silvotti ${ }^{30}$, S. Galleti $^{30}$, T. Nagel ${ }^{31}$, G. VAuclair $^{32}$, N. Dolez $^{33}$,

J. R. Fremy ${ }^{33}$, J. Perez ${ }^{34}$, J. M. Almenara ${ }^{34}$, And L. FraGa ${ }^{14}$

${ }^{1}$ Department of Physics and Astronomy, University of Delaware, Newark, DE 19716, USA; jlp@udel.edu

${ }^{2}$ Delaware Asteroseismic Research Center, Mt. Cuba Observatory, Greenville, DE 19807, USA

${ }^{3}$ Department of Astronomy, University of Texas, Austin, TX 78712, USA; mikemon@ rocky.as.utexas.edu

${ }^{4}$ Departamento de Física, Universidade Federal de Santa Catarina, C.P. 476, 88040-900 Florianópolis, SC, Brazil; ankanaan@ gmail.com

${ }^{5}$ SETI Institute, NASA Ames Research Center, Moffett Field, CA 94035, USA

${ }^{6}$ Department of Math and Science, Delaware County Community College, 901 South Media Road, Media, PA 19063, USA; dpc@udel.edu

${ }^{7}$ Department of Physics and Astronomy, University of North Carolina, 288 Phillips Hall, Chapel Hill, NC 27599, USA; clemens@ physics.unc.edu

${ }^{8}$ NRAO, Green Bank, WV 24944, USA; rachel.rosen@gmail.com

${ }^{9}$ Department of Chemistry and Physics, Georgia College and State University, Milledgeville, GA 31061, USA; agnes.kim@gcsu.edu

${ }^{10}$ Meyer Observatory and Central Texas Astronomical Society, 209 Paintbrush, Waco, TX 76705, USA; chandler@vvm.com

${ }^{11}$ Institute for Astronomy, Southwestern University, Georgetown, TX, USA; tkw@ sousthwestern.edu

${ }^{12}$ Institut für Astronomie Universität Wien, Türkenschanzstrasse 17, 1180 Vienna, Austria; gerald@ camk.edu.pl

${ }^{13}$ Department of Physics \& Space Sciences, Florida Institute of Technology, 150 West University Boulevard, Melbourne, FL 3290, USA; wood@ fit.edu

${ }^{14}$ Instituto de Fíisica UFRGS, C.P. 10501, 91501-970 Porto Alegre, RS, Brazil; kepler@ if.ufrgs.br

${ }^{15}$ Department of Physics, Astronomy and Material Science, Missouri State University and Baker Observatory, 901 S. National, Springfield, MO 65897, USA; MikeReed@missouristate.edu

${ }^{16}$ Gemini Observatory, Northern Operations Center, 670 North A'ohoku Place, Hilo, HI 96720, USA; atsuko.nittakleinman1@ gmail.com

${ }^{17}$ Las Cumbres Observatory Global Telescope Network, Inc., 6740 Cortona Drive Suite 102, Santa Barbara, CA 93117, USA; tbrown@ 1cogt.com

${ }^{18}$ Korea Astronomy and Space Science Institute, Daejeon 305-348, Republic of Korea; slkim@kasi.re.kr

${ }^{19}$ School of Chemical \& Physical Sciences, Victoria University of Wellington, P.O. Box 600, Wellington, New Zealand; denis.sullivan@vuw.ac.nz

${ }^{20}$ Lulin Observatory, National Central University, Jhongli City, Taoyuan County, Taiwan; wchen@ astro.ncu.edu.tw

${ }^{21}$ National Astronomical Observatories, Academy of Sciences, Beijing 100012, PR China; xjjiang@bao.ac.cn

${ }^{22}$ Ukrainian National Academy of Sciences, Main Astronomical Observatory, Golosiiv, Kiev 022 252650, Ukraine; sergeev@ terskol.com

${ }^{23}$ Institute of Theoretical Physics and Astronomy, Vilnius University, Vilnius, Lithuania; jr@itpa.lt

${ }^{24}$ Physical Research Laboratory, Ahmedabad 380009, India

${ }^{25}$ Mount Suhora Observatory, Cracow Pedagogical University, Ul. Podchorazych 2, 30-084 Krakow, Poland; zola@ astro1.as.ap.krakow.pl

${ }^{26}$ Astronomical Observatory, Jagiellonian University, Ul. Orla 171, 30-244 Cracow, Poland

${ }^{27}$ Konkoly Observatory, P.O. Box 67, H-1525 Budapest XII, Hungary; paparo@konkoly.hu

${ }^{28}$ Department of Physics, University of the Western Cape, Private Bag X17, Belville 7535, South Africa

${ }^{29}$ South African Astronomical Observatory, P.O. Box 9, Observatory 7935, South Africa

${ }^{30}$ INAF-Osservatorio Astronomico di Capodimonte, via Moiariello 16, 80131 Napoli, Italy

${ }^{31}$ Institut für Astronomie und Astrophysik, Universiät Tübingen, Sand 1, 72076 Tübingen, Germany; nagel@ astro.uni-tuebingen.de

${ }^{32}$ Laboratoire d'Astrophysique de Toulouse-Tarbes, Université de Toulouse, CNRS, 14 avenue Edouard Belin,

F314000 Toulouse, France; gerardv@ srvdec.obs-mip.fr

${ }^{33}$ Observatoire de Paris, LESIA, 92195 Meudon, France

${ }^{34}$ Instituto de Astrofisica de Canarias, 38200 La Laguna, Tenerife, Spain

Received 2012 January 23; accepted 2012 April 6; published 2012 May 9

\begin{abstract}
We report on an analysis of $308.3 \mathrm{hr}$ of high-speed photometry targeting the pulsating DA white dwarf EC14012-1446. The data were acquired with the Whole Earth Telescope during the 2008 international observing run XCOV26. The Fourier transform of the light curve contains 19 independent frequencies and numerous combination frequencies. The dominant peaks are 1633.907, 1887.404, and $2504.897 \mu \mathrm{Hz}$. Our analysis of the combination amplitudes reveals that the parent frequencies are consistent with modes of spherical degree $l=1$. The combination amplitudes also provide $m$ identifications for the largest amplitude parent frequencies. Our seismology analysis, which includes 2004-2007 archival data, confirms these identifications, provides constraints on additional frequencies, and finds an average period spacing of $41 \mathrm{~s}$. Building on this foundation, we present nonlinear fits to high signal-to-noise light curves from the SOAR 4.1 m, McDonald $2.1 \mathrm{~m}$, and KPNO $2 \mathrm{~m}$ telescopes. The fits indicate a time-averaged convective response timescale of $\tau_{0}=99.4 \pm 17 \mathrm{~s}$, a temperature exponent $N=85 \pm 6.2$, and an inclination angle of $\theta_{i}=32.9 \pm 3.2$. We present our current empirical map of the convective response timescale across the DA instability strip.
\end{abstract}

Key words: asteroseismology - stars: evolution - stars: individual (EC14012-1446) - stars: oscillations - white dwarfs

Online-only material: color figures 


\section{INTRODUCTION}

Stellar seismology, also known as asteroseismology, provides us with a unique tool for probing the interiors of stars, allowing us to study fundamental problems in stellar evolution such as energy transport, thermodynamics, and magnetism. White dwarfs, the evolutionary endpoint of most stars, are particularly important targets for asteroseismology. They are structurally simple: an electron degenerate carbon/oxygen core surrounded by thin non-degenerate layers of hydrogen and helium. DA white dwarfs represent $\sim 80 \%$ of all white dwarfs (Eisenstein et al. 2006), and they have a nearly pure layer of hydrogen on top of a layer of helium. DB white dwarfs lack this hydrogen layer, having a layer of nearly pure helium overlying a carbon/oxygen core. Lacking substantial nuclear reactions, white dwarfs simply cool as they age, passing through specific temperature ranges (the DBV and DAV instability strips) within which they pulsate. These pulsators are otherwise normal objects, so what we learn about their structure can be applied to the entire population of stellar remnants and further applied to our understanding of their main-sequence progenitors.

In this paper, we focus on combining asteroseismology of the DAV EC14012-1456 with nonlinear analysis of its light curve to provide an empirical description of its convection zone. Convection is one of the largest remaining sources of theoretical uncertainty in astrophysical modeling. Convection is traditionally treated through mixing length theory (MLT; Böhm-Vitense 1958). MLT is a simple, local, time-independent description first applied to stellar modeling by Biermann (1932). It describes the motions of "average" convective cells with a mean size $l=\alpha H_{p}$, where $H_{p}$ is the local pressure scale height and $\alpha$ is an adjustable free parameter. MLT is clearly incomplete; we know turbulent flows are complex and there is no reason why $\alpha$ should remain constant throughout the convection zone of a single star, and certainly not for stars of different masses, chemical compositions, or evolutionary phases. As an example for white dwarfs, Bergeron et al. (1995) and Tremblay et al. (2010) show that model parameters such as flux, line profiles, energy distribution, color indices, and equivalent widths are extremely sensitive to the assumed MLT parameterization. Bergeron et al. (1995) find systematic uncertainties ranging from $25 \%$ for effective temperatures to $11 \%$ for mass and radius. The use of MLT to treat convective energy transport in white dwarf atmospheres thus represents a significant source of physical uncertainty in calculations of their atmospheric structure. We rely on these models to supply the information about white dwarf interiors, masses, and temperatures needed to calibrate white dwarf cooling sequences. This in turn produces detailed age estimates for white dwarfs (Ruiz \& Bergeron 2001) and an estimate of the age of the Galactic disk (Winget et al. 1987; Harris et al. 2006). An observational test of MLT that leads to an improved description of convection is an important goal that will have implications beyond the study of white dwarfs.

Brickhill (1992) was the first to realize that a pulsating white dwarf's photospheric flux is modulated relative to the flux entering at the bottom of the convection zone by an amount that depends on the convection zone's thickness. The local convection zone depth is a function of the local effective

\footnotetext{
* Based on observations obtained at the Southern Astrophysical Research (SOAR) telescope, which is a joint project of the Ministerrio da Ciência, Tecnologia, e Inovação (MCTI) da República Federativa do Brasil, the U.S National Optical Astronomy Observatory (NOAO), the University of North Carolina at Chapel Hill (UNC), and Michigan State University (MSU).
}

temperature, and this varies during a pulsation cycle. The result is a distortion of the observed light curve, with narrow peaks and wider valleys (Figure 1). Convective light curve fitting exploits these nonlinearities to recover the thermal response timescale of the convection zone. Mathematical details of this technique can be found in Montgomery (2005c) and Montgomery et al. (2010b). For our purposes, applying convective light curve fitting to a target star requires three ingredients: (1) a pulsator with a nonlinear light curve, (2) precise knowledge of the star's pulsation frequencies and $(l, m)$ values, and (3) high signal-tonoise light curves for use in the actual fitting process.

Asteroseismology provides the tools to identify white dwarf pulsation $(l, m)$ values. White dwarfs are $g$-mode pulsators, and each pulsation mode can be described by a spherical harmonic of degree $l$, radial overtone $k$, and azimuthal number $m$, where $m$ takes integer values between $-l$ and $l$. Given a sufficient sample of excited pulsation modes, we can match the observed frequencies with theoretical models. An important diagnostic for $g$-mode pulsators is the mean period spacing between modes of the same $(l, m)$ but consecutive radial overtone $k$ (e.g., the $k$ and $k+1$ modes). The mean period spacing depends mainly on stellar mass. Deviations of individual spacings from this mean value provide information on the thickness of the hydrogen and/or helium layers and on the chemical profile of the core (Montgomery 2009). We note here that the actual value of the radial overtone $k$ cannot be determined observationally, but must be inferred from theoretical models.

A second diagnostic is given by the presence of multiplets. The multiplet components have the same $(k, l)$ and are further described by the azimuthal index $m$, which takes integer values between $-l$ and $l$. To first order, the frequency difference relative to the $m=0$ component of the multiplet is given by $\delta v_{k l m}=-m \Omega\left(1-C_{k l}\right)$, where $\Omega$ is the rotation period and $C_{k l}$ is a coefficient that depends on the pulsation eigenfunctions evaluated in the nonrotating case. In the high- $k$ asymptotic limit for $g$-modes, $C_{k l} \sim 1 / \ell(\ell+1)$, although models predict it to vary by $\approx 10 \%$ over the range of observed periods in EC14012. Multiplet structure is a strong indication of a mode's $l$ value. We expect a triplet for $l=1$, a quintuplet for $l=2$, and so on. The observed frequency differences (splittings) are a measure of the stellar rotation rate as sampled by a given mode. Deviations from equal frequency splitting within a single multiplet and changes in splittings from one multiplet to the next reveal information about differential rotation and magnetic field strength.

Applying convective light curve fitting to a wide sample of pulsating white dwarfs provides an empirical map of how the convective response timescale varies as a function of effective temperature, and this can be compared with theoretical models, both MLT and hydrodynamic. The Whole Earth Telescope (WET) is engaged in a long-term project to provide such a description of convection across the hydrogen atmosphere DAV ( $\left.T_{\text {eff }} \approx 11,100-12,200 \mathrm{~K}\right)$ and the helium atmosphere DBV ( $\left.T_{\text {eff }} \approx 22,000-29,000 \mathrm{~K}\right)$ instability strips. We present here our results for the DAV pulsator EC14012-1446. Our goals are three-fold: determine accurate frequency and $(l, m)$ identifications, obtain several nights of high-quality light curves, and apply convective light curve fitting to obtain EC140121446's convective response timescale. In the following, we present the resulting data set and our analysis of the Fourier transforms (FTs), discuss the identified frequencies and $(l, m)$ values, perform nonlinear light curve fits, and present the convective parameters for EC14012-1446. 


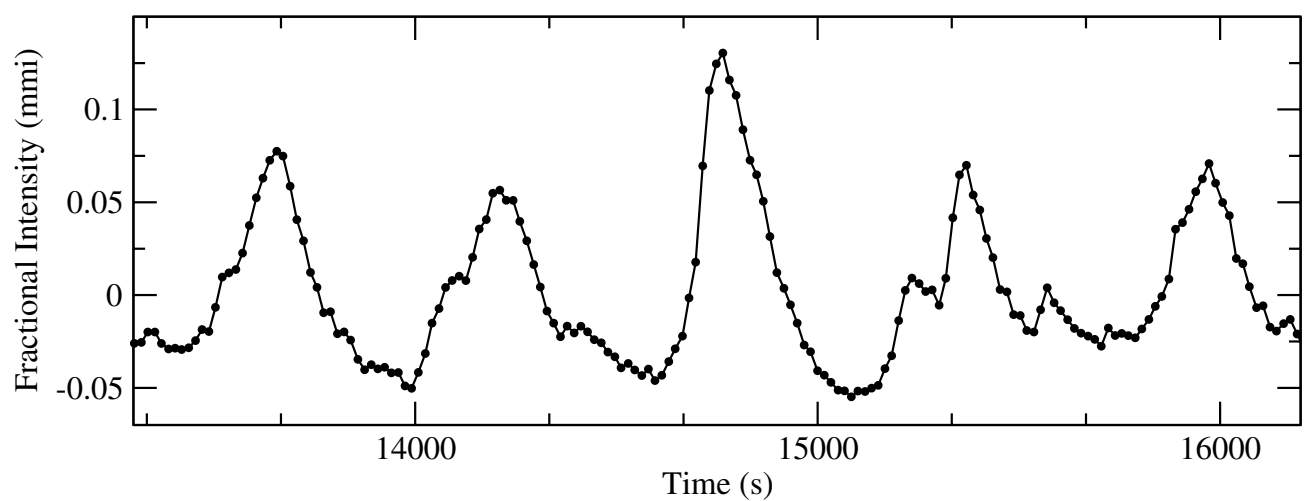

Figure 1. Portion of a high signal-to-noise SOAR light curve of EC14012-1446, showing the narrow peaks and broader valleys indicative of convective mixing. The error bars for each point are indicated by the size of the points. The dominant pulsation period is $\approx 600 \mathrm{~s}(1 \mathrm{mmi} \approx 1 \mathrm{mmag})$.

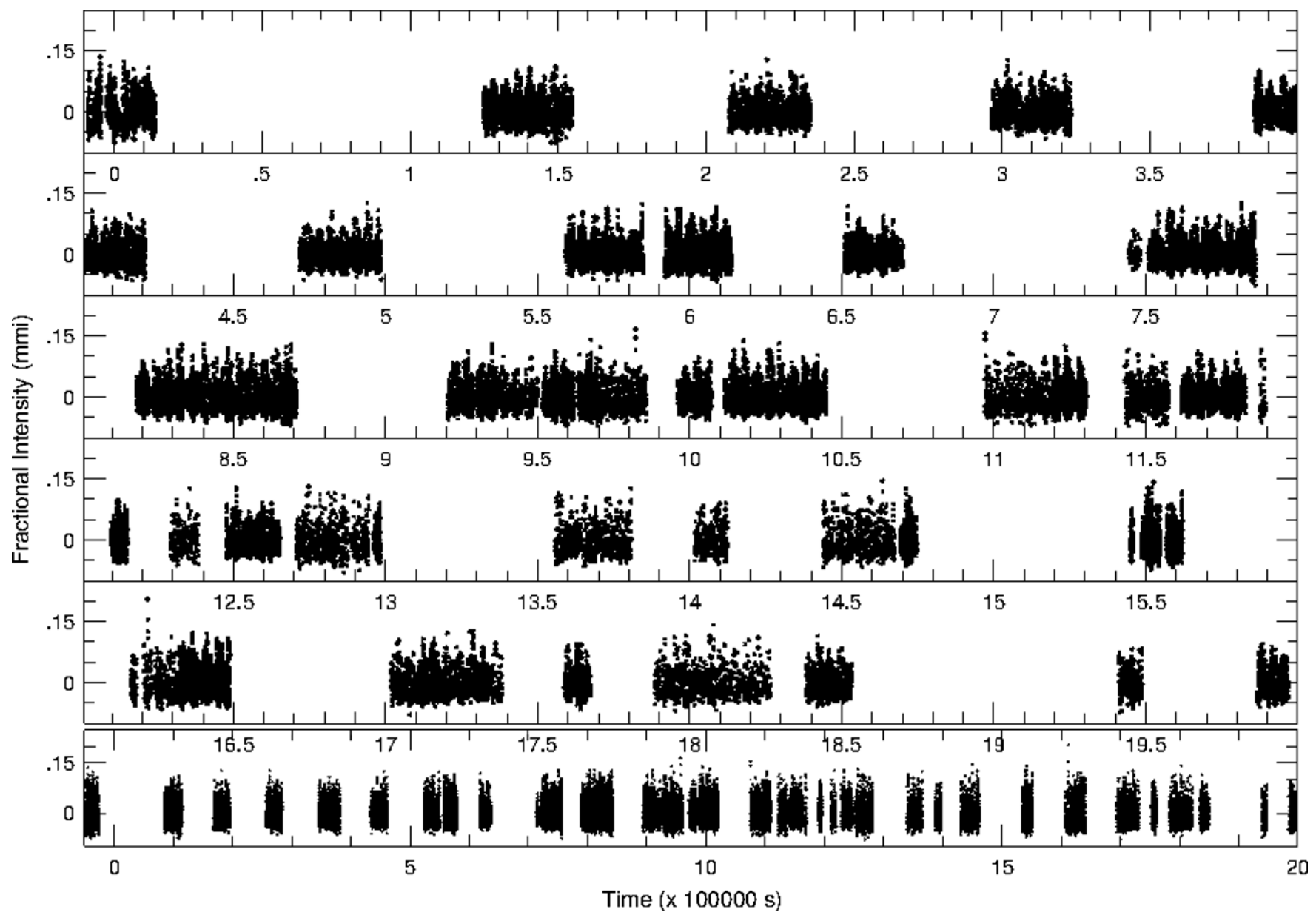

Figure 2. Final light curve of EC14012-1446 from XCOV26, representing the combination of all data listed in Table 1. The top five panels cover 400,000 s (4.63 days) each. The bottom panel displays the entire light curve, with time in days. The dominant pulsation period is $\approx 600 \mathrm{~s}$.

\section{OBSERVATIONS AND REDUCTIONS}

EC14012-1446 (WD1401-147, $B=15.67$ ) is a highamplitude, multiperiodic DAV pulsator discovered by Stobie et al. (1995) and observed extensively by Handler et al. (2008). Our XCOV26 observations span 2008 March 25 to April 29, achieving $80 \%$ coverage during the central five days of the run (Figure 2). Twenty-seven telescopes distributed around the globe participated in XCOV26, 15 of which contributed a total of 71 runs (Table 1) on EC14012-1446. The observations were obtained with different CCD photometers, each with distinct effective bandpasses. We minimize the bandpass issues by using
CCDs with similar detectors where possible and employing a red cutoff filter (BG40 or S8612) to normalize wavelength response and reduce extinction effects.

Standard procedure for a WET run calls for observers to transfer raw images and calibration files to WET headquarters for analysis at the end of each night. CCD data reduction follows the steps outlined in Provencal et al. (2009). We corrected each image for bias and thermal noise, and normalized by the flat field. Aperture photometry using the IRAF photometry pipeline described by Kanaan et al. (2002) was performed on each image, utilizing a range of aperture sizes for the target and selected comparison stars. We used the WQED pipeline (Thompson 
Table 1

Journal of XCOV26 Observations EC14012-1446

\begin{tabular}{|c|c|c|c|c|}
\hline Run Name & $\begin{array}{l}\text { Telescope } \\
\text { (m) }\end{array}$ & Detector & Date & $\begin{array}{c}\text { Length } \\
\text { (hr) }\end{array}$ \\
\hline hawa20080325-09 & Hawaii 2.2 & E2V ccd47-10 & 2008 Mar 25 & 6.2 \\
\hline saao20080326-21 & SAAO 1.0 & UCT CCD & 2008 Mar 26 & 5.9 \\
\hline tene20080327-20 & Tenerife 0.8 & TK1024 & 2008 Mar 27 & 3.0 \\
\hline saao20080327-20 & SAAO 1.0 & UCT CCD & 2008 Mar 27 & 7.5 \\
\hline saao20080328-20 & SAAO 1.0 & UCT CCD & 2008 Mar 28 & 7.3 \\
\hline saao20080329-20 & SAAO 1.0 & UCT CCD & 2008 Mar 29 & 7.2 \\
\hline tene20080330-03 & Tenerife 0.8 & TK1024 & 2008 Mar 30 & 2.9 \\
\hline saao20080330-20 & SAAO 1.0 & UTC CCD & 2008 Mar 30 & 7.5 \\
\hline saao20080331-20 & SAAO 1.0 & UTC CCD & 2008 Mar 31 & 7.1 \\
\hline mcdo20080401-05 & McDonald 2.1 & E2V ccd57-10 & 2008 Apr 1 & $6.1^{*}$ \\
\hline saao20080401-20 & SAAO 1.0 & UTC CCD & 2008 Apr 1 & 5.4 \\
\hline loia20080403-00 & Loia 1.52 & EEV $1300 \times 1340 B$ & 2008 Apr 3 & 1.1 \\
\hline ctio20080403-01 & CTIO 0.9 & Tek $2 \mathrm{~K}_{3}$ & 2008 Apr 3 & 7.5 \\
\hline soar20080403-02 & SOAR 4.1 & $2 \mathrm{~K} \times 4 \mathrm{~K}$ MIT/Lincoln Lab CCD & 2008 Apr 3 & $7.6^{*}$ \\
\hline sara20080403-05 & SARA 1.0 & $\mathrm{CCD}$ & 2008 Apr 3 & 6.3 \\
\hline mcdo20080403-08 & McDonald 2.1 & E2V ccd57-10 & 2008 Apr 3 & 3.0 \\
\hline saao20080403-20 & SAAO 1.0 & UCT CCD & 2008 Apr 3 & 6.8 \\
\hline salt20080403-21 & SALT 10.0 & $\mathrm{CCD}$ & 2008 Apr 3 & 1.0 \\
\hline soar20080404-01 & SOAR 4.1 & CCD & 2008 Apr 4 & $7.7^{*}$ \\
\hline salt20080404-02 & SALT 10.0 & E2V 44-82 & 2008 Apr 4 & 0.7 \\
\hline ctio20080404-02 & CTIO 0.9 & $\mathrm{Tek} 2 \mathrm{~K}_{3}$ & 2008 Apr 4 & 7.0 \\
\hline mcdo20080404-05 & McDonald 2.1 & E2V ccd57-10 & 2008 Apr 4 & $5.4^{*}$ \\
\hline sara20080404-10 & SARA 1.0 & $\mathrm{CCD}$ & 2008 Apr 4 & 0.6 \\
\hline saao20080404-21 & SAAO 1.0 & UTC CCD & 2008 Apr 4 & 6.4 \\
\hline salt20080404-21 & SALT 10.0 & E2V 44-82 & 2008 Apr 4 & 1.0 \\
\hline soar20080405-01 & SOAR 4.1 & $2 \mathrm{~K} \times 4 \mathrm{~K}$ MIT/Lincoln Lab CCD & 2008 Apr 5 & $4.7^{*}$ \\
\hline salt20080405-02 & SALT 10.0 & E2V 44-82 & 2008 Apr 5 & 1.0 \\
\hline ctio20080405-03 & CTIO 0.9 & Tek $2 \mathrm{~K}_{3}$ & 2008 Apr 5 & 6.3 \\
\hline mcdo20080405-09 & McDonald 2.1 & E2V ccd57-10 & 2008 Apr 5 & 1.7 \\
\hline mtjo20080405-11 & Mt. John 1.0 & E2V ccd57-10 & 2008 Apr 5 & 3.3 \\
\hline boao20080405-14 & BOAO 1.8 & SITe SI424AB CCD & 2008 Apr 5 & 4.4 \\
\hline salt20080405-21 & SALT 10.0 & E2V 44-82 & 2008 Apr 5 & 1.0 \\
\hline saao20080405-22 & SAAO 1.0 & UTC CCD & 2008 Apr 5 & 3.1 \\
\hline salt20080406-01 & SALT 10.0 & E2V 44-82 & 2008 Apr 6 & 1.0 \\
\hline soar20080406-02 & SOAR 4.1 & $2 \mathrm{~K} \times 4 \mathrm{~K}$ MIT/Lincoln Lab CCD & 2008 Apr 6 & $7.3^{*}$ \\
\hline mcdo20080406-09 & McDonald 2.1 & E2V ccd57-10 & 2008 Apr 6 & 2.0 \\
\hline ctio20080407-02 & CTIO 0.9 & Tek $2 \mathrm{~K}_{3}$ & 2008 Apr 6 & 6.2 \\
\hline mcdo20080407-08 & McDonald 2.1 & $\mathrm{E} 2 \mathrm{~V} \operatorname{ccd} 57-10$ & 2008 Apr 7 & 3.1 \\
\hline boao20080407-15 & BOAO 1.8 & SITe SI424AB CCD & 2008 Apr 7 & 4.0 \\
\hline saao20080407-20 & SAAO 1.0 & UTC CCD & 2008 Apr 7 & 5.9 \\
\hline salt20080407-20 & SALT 10.0 & E2V $44-82$ & 2008 Apr 7 & 1.0 \\
\hline salt20080408-01 & SALT 10.0 & E2V 44-82 & 2008 Apr 8 & 1.0 \\
\hline ctio20080408-02 & CTIO 0.9 & Tek $2 \mathrm{~K}_{3}$ & 2008 Apr 8 & 1.8 \\
\hline mcdo20080408-09 & McDonald 2.1 & E2V ccd57-10 & 2008 Apr 8 & 1.5 \\
\hline luli20080408-14 & Lulin 1.0 & E2V CCD36-40 & 2008 Apr 8 & 2.6 \\
\hline saao20080408-20 & SAAO 1.0 & UTC CCD & 2008 Apr 8 & 4.9 \\
\hline ctio20080409-02 & CTIO 0.9 & Tek2 $\mathrm{K}_{3}$ & 2008 Apr 9 & 6.6 \\
\hline mcdo20080409-09 & McDonald 2.1 & E2V ccd57-10 & 2008 Apr 9 & 0.6 \\
\hline ctio20080410-02 & CTIO 0.9 & Tek2 $\mathrm{K}_{3}$ & 2008 Apr 10 & 6.9 \\
\hline boao20080410-14 & BOAO 1.8 & SITe SI424AB CCD & 2008 Apr 10 & 3.0 \\
\hline ctio20080411-02 & CTIO 0.9 & Tek $2 \mathrm{~K}_{3}$ & 2008 Apr 11 & 6.6 \\
\hline mcdo20080411-09 & McDonald 2.1 & E2V ccd57-10 & 2008 Apr 11 & 1.5 \\
\hline mcdo20080412-06 & McDonald 2.1 & E2V ccd57-10 & 2008 Apr 12 & 0.3 \\
\hline kpno20080412-07 & KPNO 2.0 & E2V ccd47-10 & 2008 Apr 12 & 3.7 \\
\hline ctio20080413-02 & CTIO 0.9 & Tek $2 \mathrm{~K}_{3}$ & 2008 Apr 13 & 4.4 \\
\hline mcdo20080413-06 & McDonald 2.1 & E2V ccd57-10 & 2008 Apr 13 & 4.7 \\
\hline ctio20080414-02 & CTIO 0.9 & Tek $2 \mathrm{~K}_{3}$ & 2008 Apr 14 & 7.1 \\
\hline kpno20080414-04 & KPNO 2.0 & E2V ccd47-10 & 2008 Apr 14 & 2.4 \\
\hline lash20080414-08 & Las Cumbres 2.0 & E2V CCD42-40 & 2008 Apr 14 & 3.4 \\
\hline mcdo20080414-09 & McDonald 2.1 & E2V ccd57-10 & 2008 Apr 14 & 1.7 \\
\hline luli20080414-17 & Lulin 1.0 & E2V CCD36-40 & 2008 Apr 14 & 2.4 \\
\hline ctio20080415-02 & CTIO 0.9 & Tek $2 \mathrm{~K}_{3}$ & 2008 Apr 15 & 6.6 \\
\hline lash20080415-09 & Las Cumbres 2.0 & E2V CCD $42-40$ & 2008 Apr 15 & 3.8 \\
\hline luli20080415-16 & Lulin 1.0 & E2V ccd36-40 & 2008 Apr 15 & 4.3 \\
\hline mabu20080415-20 & Mt. Abu 1.2 & TEK CCD & 2008 Apr 15 & 1.3 \\
\hline
\end{tabular}


Table 1

(Continued)

\begin{tabular}{lccc}
\hline \hline Run Name & $\begin{array}{c}\text { Telescope } \\
(\mathrm{m})\end{array}$ & Detector & $\begin{array}{c}\text { Date } \\
(\mathrm{hr})\end{array}$ \\
\hline mabu20080416-20 & Mt. Abu 1.2 & TEK CCD & 2008 Apr 16 \\
kpno20080417-09 & KPNO 2.0 & E2V ccd47-10 & 2008 Apr 17 \\
kpno20080418-07 & KPNO 2.0 & E2V ccd47-10 & 2008 Apr 18 \\
braz20080426-04 & WN106 CCD 1.6 & WI106 CCD & 2008 Apr 26 \\
braz20080427-05 & LNA 1.6 & WI106 CCD & 2008 Apr 27 \\
braz20080428-04 & LNA 1.6 & WI106 CCD & 2008 Apr 28 \\
braz20080429-05 & LNA 1.6 & 2008 Apr 29 & 2.1 \\
\hline
\end{tabular}

Note. ${ }^{*}$ High signal-to-noise light curves used for light curve fitting. All other observations were used to obtain accurate frequency information.

\& Mullally 2009) to examine each nightly light curve for photometric quality, remove outlying points, divide by a suitable comparison star, and correct for differential extinction. Our observational technique is therefore not sensitive to oscillations longer than a few hours. The result is light curves with amplitude variations represented as fractional intensity (mmi). The unit is a linear representation of the fractional intensity of modulation $(1 \mathrm{mmi} \approx 1 \mathrm{mmag})$. We present our FTs in units of modulation amplitude $\left(1 \mathrm{mma}=1 \times 10^{-3} \mathrm{ma}=0.1 \%=1 \mathrm{ppt}\right)$.

The final reduction step combines the individual light curves to produce the complete light curve for EC14012-1446. We assume EC14012-1446 oscillates about a mean light level. This assumption allows us to carefully assess overlapping segments from different telescopes and identify and correct any vertical offsets. As discussed in detail in Provencal et al. (2009), we find no significant differences between the noise levels of amplitude spectra using (1) the combination of all light curves including overlapping segments from different telescopes, (2) the combination of light curves where we retain only higher signal-to-noise observations in overlapping segments, and (3) combining all light curves using data weighted by telescope aperture.

The final XCOV26 light curve contains $308.3 \mathrm{hr}$ of highspeed photometry. Our coverage is not complete, and this incompleteness produces spectral leakage in the amplitude spectrum. To quantify this, we sampled a single sinusoid using exactly the same times as the original data. The resulting amplitude spectrum, or "spectral window," is the pattern produced in the XCOV26 FT by a single frequency. The FT and spectral window of the complete light curve are given in Figure 3.

\section{FREQUENCY IDENTIFICATION}

\subsection{Stability}

Following Provencal et al. (2009), we begin by examining the stability of EC14012-1446's pulsation amplitudes and frequencies throughout the run. Amplitude and/or frequency variations produce artifacts in FTs, and greatly complicate the identification of intrinsic pulsation frequencies (Kepler et al. 2003). We divide the data set into three chunks, each spanning $\approx 185 \mathrm{hr}$ (7.8 days). The FT of each chunk is given in Figure 4. Several of the dominant frequencies are consistent to within measurement error, but we do find evidence of amplitude variations in a number of frequencies.

We calculated spectrograms for the five largest amplitude frequencies to further explore the nature of these amplitude variations (Figure 5). A spectrogram quantifies the behavior of frequencies and amplitudes as a function of time. Our spectrograms are generated by dividing the total light curve into multiple 5 day segments, each of which overlap by 4.95 days. The FT of each segment is a measurement of frequencies and amplitudes centered on a specific time. Each panel in Figure 5 is an amalgam, where each segment FT corresponds to a vertical line. The $x$-axis is time, the $y$-axis is frequency, and amplitude is represented by color and is normalized to an amplitude of 1. The bottom panel in Figure 5 gives the pattern generated by a single sinusoid sampled with exactly the same times as the segment light curves (a time-dependent spectral window). The results show a slow $18 \sigma$ increase in the amplitude of the dominant $1633.907 \mu \mathrm{Hz}(612 \mathrm{~s})$ peak, from $\approx 22$ to $\approx 31 \mathrm{mma}$, over the course of the run. We also find a similar increase in the $1548.146 \mu \mathrm{Hz}$ (645 s) peak. The $2308 \mu \mathrm{Hz}$ (433 s) peak shows a $25 \%$ decrease in amplitude during the middle of the run and a large apparent decrease at the end of the run. The amplitudes of the remaining two frequencies are consistent to within $3 \sigma$.

\subsection{The XCOV26 Fourier Transform}

Armed with amplitude stability information for the five largest peaks, we are ready to take a careful look at EC140121446's XCOV26 FT. We use Period04 (Lenz \& Breger 2005) for Fourier analysis and nonlinear least-squares fitting to select the statistically significant peaks in the XCOV26 FT. As detailed in Provencal et al. (2009), we adopt the criterion that a peak must have an amplitude at least four times greater than the mean noise level in the given frequency range. We define "noise" as the frequency-dependent mean amplitude after prewhitening by the dominant frequencies. This is a conservative estimate, as it is impossible to ensure that all of the "real" frequencies are removed when calculating the noise level. This is certainly true for EC14012-1446, where the peaks above $\approx 3000 \mu \mathrm{Hz}$ are mainly combination frequencies. Figure 6 displays the mean amplitude, specified as the square root of the simple mean power using a boxcar of $100 \mu \mathrm{Hz}$, after prewhitening by 62 frequencies, as a function of frequency. Our noise is somewhat frequency dependent, but is near $0.2 \mathrm{mma}$.

To confirm our uncertainty estimates, we calculated Monte Carlo simulations using the routine provided in Period04. This routine generates a set of light curves using the original times, the fitted frequencies and amplitudes, and added Gaussian noise. A least-squares fit is performed on each simulated light curve, with the distribution of fit parameters giving the uncertainties. Our Monte Carlo results are consistent with our mean amplitude noise estimates of $\approx 0.2 \mathrm{mma}$.

Our frequency selection procedure involves identifying the largest amplitude resolved peak in the FT, fitting a sinusoid with that frequency to the data set, subtracting the fit from the light curve, recomputing the FT, examining the residuals, and repeating the process until no significant power remains. 


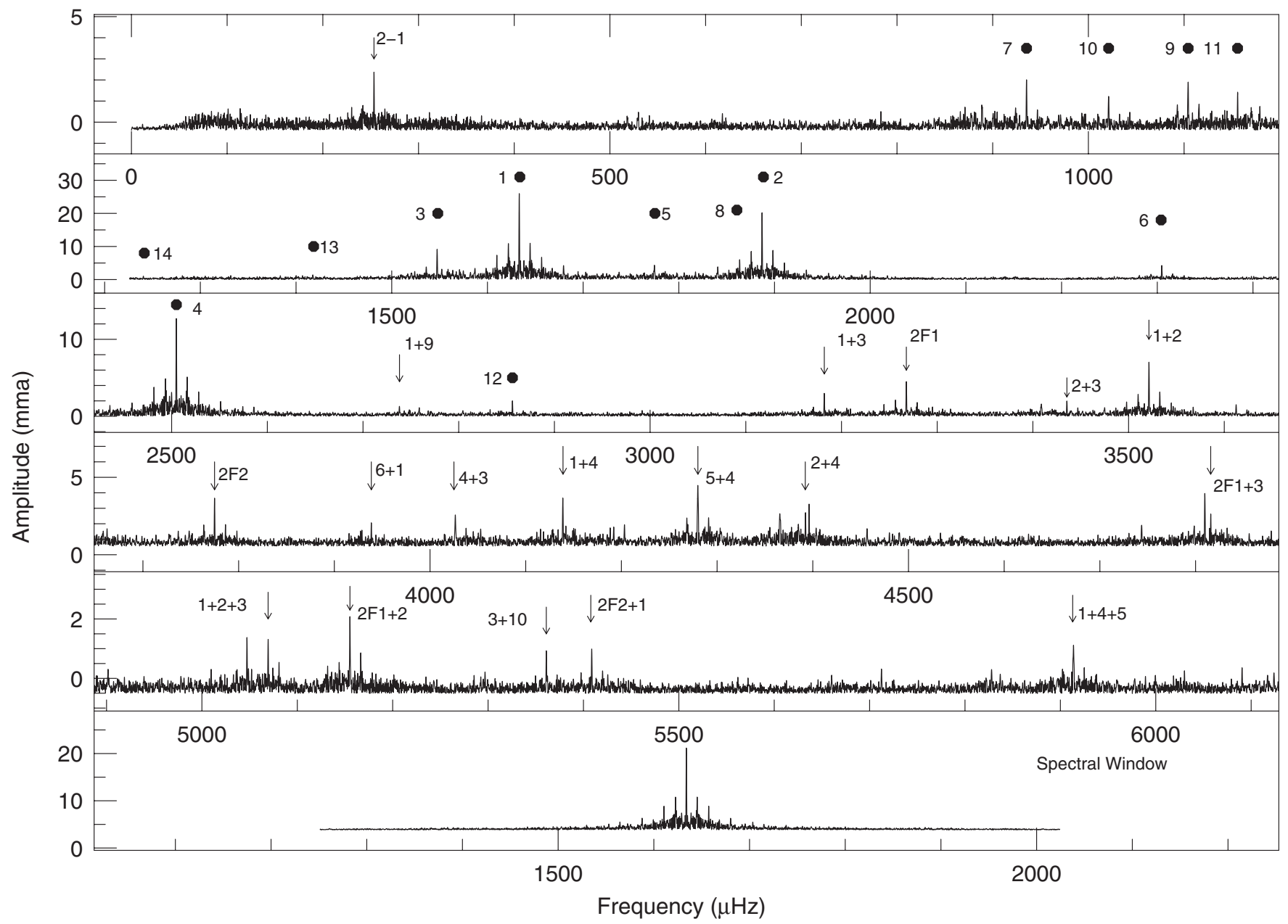

Figure 3. FT of the 2008 EC14012-1446 photometry observations (note vertical scale in each panel). Solid dots label 14 modes containing 19 independent frequencies. Arrows indicate a sample of combination frequencies (for example $\mathrm{F} 1+\mathrm{F} 2$ ). Unlabeled peaks are second-order (for example $\mathrm{F} 1+\mathrm{F} 2$ ) and third-order (for example $\mathrm{F} 1+\mathrm{F} 2+\mathrm{F} 3)$ combinations. The spectral window is plotted in the last panel. Tables 2 and 3 list exact frequency identifications.

This technique, known as prewhitening, must be employed with an abundance of caution, especially since we are aware of amplitude and/or frequency modulation in our data set. The modulation will create artifacts that masquerade as additional frequencies. To illustrate, let us examine the region of dominant power at $1633.907 \mu \mathrm{Hz}$ (Figure 7). Comparison of the original FT (top panel) with the spectral window (bottom panel) demonstrates that most of the signal is concentrated at $1633.907 \mu \mathrm{Hz}$. We fit a sinusoid to the data to determine frequency, amplitude, and phase, and subtract the result from the original light curve. The second panel of Figure 7 shows the prewhitened FT. Careful examination reveals two residual peaks (at 1633.450 and $1624.015 \mu \mathrm{Hz}$ identified with arrows) that are clearly not components of the spectral window. A third peak at $1622 \mu \mathrm{Hz}$ is part of a window pattern associated with $1633.450 \mu \mathrm{Hz}$, but the alias pattern is asymmetric. Panel 3 shows the results of prewhitening by a simultaneous fit of 1633.907 and $1633.450 \mu \mathrm{Hz}$. Both frequencies are removed, and $1622 \mu \mathrm{Hz}$ is diminished, leaving $1624.015 \mu \mathrm{Hz}$. We next subtract a simultaneous fit of $1633.907,1633.450$, and $1624.015 \mu \mathrm{Hz}$, with the results displayed in panel 4 of Figure 7. No significant power remains. However, a red flag is raised: the separation between 1633.907 and $1633.450 \mu \mathrm{Hz}$ is $0.457 \mu \mathrm{Hz}$, the inverse of which is the run length. The $1633.450 \mu \mathrm{Hz}$ peak is a manifestation of the amplitude changes observed in Figure 5 and is not included in our final frequency list.

Similar analyses of several other frequencies turned out to be unexpectedly complex. The power at $1775 \mu \mathrm{Hz}$ (labeled " 5 " in Figure 3) is unresolved. Figure 8 establishes that prewhitening this peak requires four closely spaced frequencies, with frequency differences very similar to the inverse of the run length. This is a clear signature of amplitude and/or frequency instability. Figure 9 shows the spectrogram of this region using the same criteria as the spectrograms in Figure 5. Unlike the slow amplitude increase observed with the $1633 \mu \mathrm{Hz}$ peak, the $1775 \mu \mathrm{Hz}$ peak undergoes remarkably sudden variations, on timescales of a few days. We also find a decrease in its frequency of $1.2 \mu \mathrm{Hz}$ $(5 \sigma)$ over the course of the run. A second region of power at $1860 \mu \mathrm{Hz}$ displays similar behavior. Both peaks are labeled in Table 2.

Our final identifications result from a simultaneous nonlinear least-squares fit of 19 independent frequencies, amplitudes, and phases as well as 68 combination frequencies. Combination frequencies are fixed with respect to their parents but their amplitudes and phases are allowed to vary. Table 2 lists 19 identified independent frequencies, with consideration to those exhibiting amplitude and/or frequency modulation. The list includes a doublet with a splitting of $9.9 \mu \mathrm{Hz}$ associated with the 


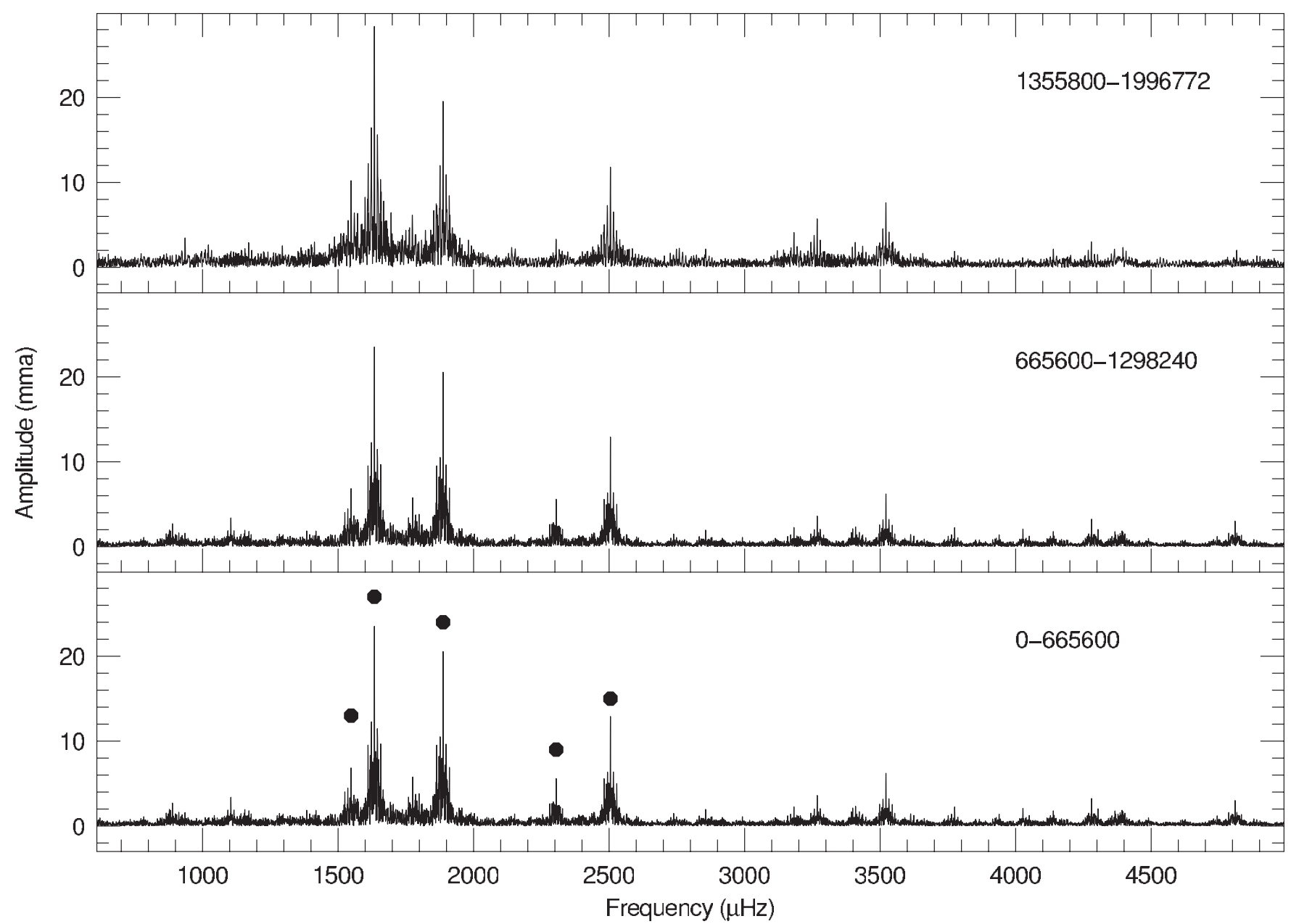

Figure 4. FTs of the EC14012-1446 data set subdivided into three chunks of $\approx 185 \mathrm{hr}$ each. The labels in the right of each panel give the time segment covered by each chunk. The black points in the bottom panel identify the five largest amplitude modes. Some changes in each FT can be explained as differences in the window structure and effective resolution for each chunk. In particular, the final chunk has the least data coverage. We do find evidence of amplitude and/or frequency variations during the run. All peaks with frequencies higher than $\approx 3000 \mu \mathrm{Hz}$ are combination peaks.

1633.907 $\mu \mathrm{Hz}$ peak (labeled 1 in Figure 3), a triplet at $1887 \mu \mathrm{Hz}$ with an average splitting of $3.8 \mu \mathrm{Hz}$ (labeled 2 in Figure 3), and a second doublet with a splitting of $3.1 \mu \mathrm{Hz}$ at $2504 \mu \mathrm{Hz}$ (labeled 4 in Figure 3). Table 3 presents the largest amplitude combination frequencies (see Section 4.1).

\section{MODE IDENTIFICATION}

Our ultimate goal is to use EC14012-1446's nonlinear light curve to extract the star's convective parameters. The requirements for convective light curve fitting include precise knowledge of the target star's frequencies and amplitudes, as well as a good idea of the $(l, m)$ values for these frequencies. The parameter space to be explored to determine the best convective light curve fit is greatly constrained by knowledge of $(l, m)$ indices for the excited pulsations. Montgomery's first application of this technique focused on the DA pulsators G29-38 and GD154 (Montgomery 2005a). In the data sets used, these objects were dominated by a single pulsation mode, so the number of potential $(l, m)$ identifications was small enough that all possibilities could be explored. Montgomery et al. (2010b) recently expanded this work to the multiperiodic pulsator GD358, a well-studied object with detailed $(l, m)$ identifications of its pulsation modes (Provencal et al. 2009; Metcalfe et al. 2000; Winget et al. 1994). While XCOV26 provided pre- cise frequencies and amplitudes for the modes in EC14012-1446 (see Table 2), we lack prior $(l, m)$ identifications. Even limiting ourselves to $l=1$ and 2, as these are the spherical degrees most often observed in pulsating white dwarfs, yields a total number of possible combinations of order $(2 l+1)^{19}$, a very large number indeed. In this section, we will rely on analysis of the combination frequencies and the support of asteroseismology to constrain $(l, m)$ identifications in EC14012-1446.

\subsection{Combination Frequencies}

Combination frequencies are typically observed in the FTs of moderate to large amplitude pulsators (e.g., Provencal et al. 2009; Dolez et al. 2006; Handler et al. 2002); they are identified by their relationships, which must be exact within measurement error. Combination frequencies can be integer multiples of a single parent (harmonics) or sums (or differences) of any two modes. These frequencies are not independent, but result from nonlinear effects most likely associated with the surface convection zone (Brickhill 1992; Brassard et al. 1995; Wu 2001; Ising \& Koester 2001). Wu (2001) shows that the observed amplitudes of combination frequencies depend on geometric factors such as the $(l, m)$ indices of the parent(s) and the inclination of the pulsation axis to the line of sight. EC140121446's FT contains a rich distribution of combinations that 


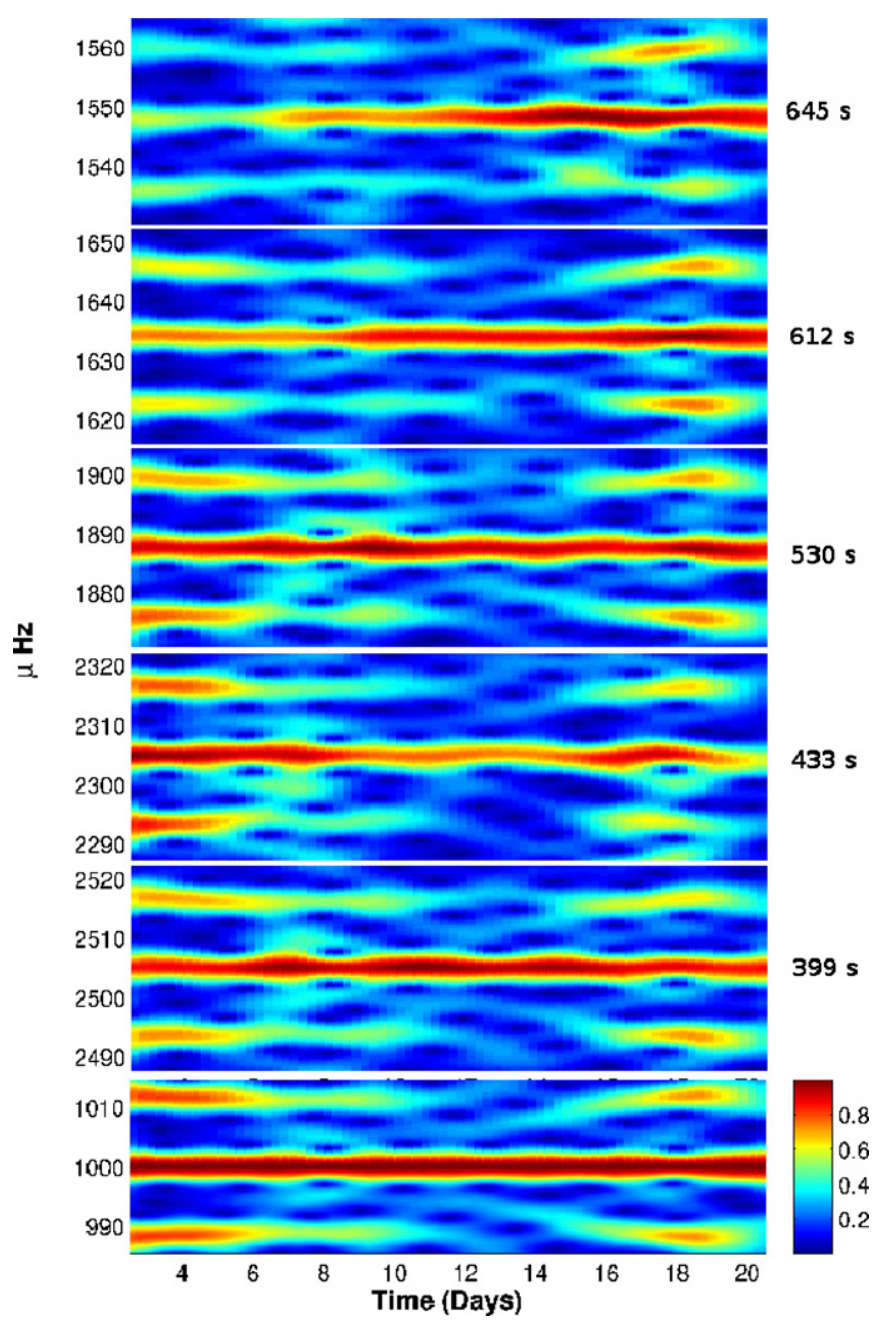

Figure 5. Spectrogram of the five largest amplitude modes in EC14012-1446's FT, arranged in order of decreasing period. The bottom panel gives the timevarying spectral window for a sample frequency at $1000 \mu \mathrm{Hz}$ (the pattern given by a single sinusoid sampled at exactly the same times as the data). Each panel is normalized to an amplitude of 1 . We find a slow amplitude increase in the $1633.907 \mu \mathrm{Hz}(612 \mathrm{~s})$ and $1548.146 \mu \mathrm{Hz}(645 \mathrm{~s})$ peaks. In addition, the $2308 \mu \mathrm{Hz}$ (433 s) peak shows a decrease in amplitude during the middle of the run.

(A color version of this figure is available in the online journal.)

involve 13 of the 19 independent frequencies listed in Table 2. Our goal is to exploit the geometric sensitivities to provide $(l, m)$ constraints for these frequencies.

$\mathrm{Wu}$ (2001) and Yeates et al. (2005) lay the foundations for our analysis by describing analytical expressions for the predicted amplitudes and phases of combination frequencies. These quantities depend on the inclination angle $\theta$ of the pulsation axis to the line of sight, the $(l, m)$ indices of the parent mode(s), the amplitudes of the parent mode(s), and parameters describing the convection zone. To minimize the dependence on the convective parameters and focus on the geometric factors $(l, m)$ and $\theta$, our analysis follows Yeates et al. (2005) and considers only combination frequencies that are the sum of two parent frequencies. We combine a genetic algorithm (Charbonneau 1995) with Wu's (2001) formulae, and incorporate an improved treatment of limb darkening taken directly from the models of Koester (Montgomery et al. 2010b). For a single run of the code, the best simultaneous fit to the observed amplitudes of the parent and combination frequencies utilizes multiple generations and minimizes the root-mean-
Table 2

Table of XCOV27 Independent Frequencies

\begin{tabular}{lccrrr}
\hline \hline ID & $\begin{array}{c}\text { Frequency } \\
(\mu \mathrm{Hz})\end{array}$ & $\begin{array}{c}\text { Period } \\
(\mathrm{s})\end{array}$ & $\begin{array}{c}\text { Amplitude } \\
(\mathrm{mma})\end{array}$ & $\mathrm{S} / \mathrm{N}$ & Notes \\
\hline 7 & $935.3799 \pm 0.002$ & 1069.085 & $2.7 \pm 0.1$ & 9 & \\
10 & $1021.139 \pm 0.002$ & 979.299 & $1.7 \pm 0.1$ & 8 & \\
9 & $1104.252 \pm 0.001$ & 905.591 & $2.2 \pm 0.2$ & 10 & \\
11 & $1155.925 \pm 0.002$ & 865.108 & $1.9 \pm 0.2$ & 8 & \\
14 & $1241.403 \pm 0.002$ & 805.540 & $1.2 \pm 0.2$ & 5 & \\
13 & $1418.369 \pm 0.002$ & 705.035 & $1.2 \pm 0.2$ & 5 & \\
$3 \mathrm{a}$ & $1521.575 \pm 0.002$ & 657.214 & $2.2 \pm 0.2$ & 9 & $\mathrm{a}$ \\
3 & $1548.146 \pm 0.001$ & 645.933 & $7.9 \pm 0.1$ & 32 & \\
$1 \mathrm{a}$ & $1624.015 \pm 0.003$ & 615.758 & $3.1 \pm 0.2$ & 13 & $\mathrm{a}$ \\
1 & $1633.907 \pm 0.001$ & 612.030 & $25.7 \pm 0.1$ & 104 & \\
5 & $1774.989 \pm 0.100$ & 563.384 & $7.2 \pm 0.3$ & 9 & $\mathrm{~b}$ \\
8 & $1860.248 \pm 0.400$ & 537.563 & $6.4 \pm 0.4$ & 11 & $\mathrm{~b}$ \\
$2 \mathrm{a}$ & $1883.555 \pm 0.003$ & 530.911 & $1.5 \pm 0.2$ & 5 & $\mathrm{a}$ \\
2 & $1887.404 \pm 0.001$ & 529.828 & $20.7 \pm 0.1$ & 79 & \\
$2 \mathrm{~b}$ & $1891.142 \pm 0.002$ & 528.781 & $3.8 \pm 0.2$ & 14 & $\mathrm{a}$ \\
6 & $2304.745 \pm 0.001$ & 433.887 & $4.7 \pm 0.1$ & 23 & $\mathrm{~b}$ \\
4 & $2504.897 \pm 0.001$ & 399.218 & $12.7 \pm 0.1$ & 71 & \\
$4 \mathrm{a}$ & $2508.060 \pm 0.002$ & 398.715 & $2.1 \pm 0.2$ & 12 & $\mathrm{a}$ \\
12 & $2856.155 \pm 0.002$ & 350.121 & $2.0 \pm 0.2$ & 9 & \\
\hline & & & & &
\end{tabular}

Notes.

${ }^{a}$ Frequency IDs with small letters indicate components of the same multiplet.

${ }^{\mathrm{b}}$ Frequencies displaying long timescale amplitude modulation.

Table 3

Table of Combination Frequencies

\begin{tabular}{lcccc}
\hline \hline Frequency & $\begin{array}{c}\text { Period } \\
(\mu \mathrm{Hz})\end{array}$ & $\begin{array}{c}\text { Amplitude } \\
(\mathrm{s})\end{array}$ & $\begin{array}{c}\mathrm{S} / \mathrm{N} \\
(\mathrm{mma})\end{array}$ & Parents \\
\hline $253.466 \pm 0.002$ & 3945.302 & $2.9 \pm 0.2$ & 9 & $2-1$ \\
$2569.286 \pm 0.002$ & 350.121 & $0.6 \pm 0.2$ & 4 & $1+7$ \\
$2738.153 \pm 0.002$ & 365.210 & $0.9 \pm 0.2$ & 4 & $1+9$ \\
$3096.255 \pm 0.002$ & 322.971 & $0.6 \pm 0.2$ & 3 & $2 \mathrm{f3}$ \\
$3182.045 \pm 0.002$ & 314.263 & $2.7 \pm 0.2$ & 12 & $1+3$ \\
$3267.794 \pm 0.002$ & 306.015 & $4.3 \pm 0.2$ & 13 & $2 \mathrm{f} 1$ \\
$3408.939 \pm 0.002^{\mathrm{a}}$ & 293.346 & $1.6 \pm 0.2$ & 5 & $6+5$ \\
$3409.912 \pm 0.002^{\mathrm{a}}$ & 293.263 & $1.7 \pm 0.2$ & 4 & $1+5$ \\
$3435.538 \pm 0.002$ & 291.075 & $2.0 \pm 0.2$ & 6 & $2+3$ \\
$3494.064 \pm 0.002^{\mathrm{a}}$ & 286.152 & $0.8 \pm 0.2$ & 4 & $1+8$ \\
$3521.306 \pm 0.002$ & 283.986 & $7.0 \pm 0.2$ & 33 & $1+2$ \\
$3525.050 \pm 0.002$ & 283.684 & $0.9 \pm 0.2$ & 5 & $1+2 \mathrm{~b}$ \\
$3774.810 \pm 0.001$ & 264.914 & $2.1 \pm 0.2$ & 12 & $2 \mathrm{f} 2$ \\
$3938.646 \pm 0.002$ & 253.894 & $0.9 \pm 0.2$ & 5 & $1+6$ \\
$4026.445 \pm 0.002$ & 248.358 & $1.4 \pm 0.2$ & 7 & $4+3 \mathrm{a}$ \\
$4053.043 \pm 0.002$ & 246.728 & $0.7 \pm 0.2$ & 4 & $3+4$ \\
$4138.800 \pm 0.002$ & 241.616 & $2.0 \pm 0.2$ & 8 & $1+4$ \\
$4192.150 \pm 0.002$ & 238.541 & $0.7 \pm 0.2$ & 4 & $2+6$ \\
$4279.907 \pm 0.002^{\mathrm{a}}$ & 233.650 & $2.6 \pm 0.2$ & 10 & $4+5$ \\
$4365.040 \pm 0.002^{\mathrm{a}}$ & 234.464 & $0.9 \pm 0.2$ & 4 & $4+8$ \\
$4365.608 \pm 0.002^{\mathrm{a}}$ & 229.063 & $1.3 \pm 0.2$ & 5 & $4+8$ \\
$4392.348 \pm 0.002$ & 227.669 & $1.5 \pm 0.2$ & 7 & $2+4$ \\
$4396.055 \pm 0.002$ & 227.477 & $1.8 \pm 0.2$ & 8 & $2 \mathrm{~b}+4$ \\
$4743.561 \pm 0.002$ & 210.812 & $0.9 \pm 0.2$ & 5 & $2+12$ \\
$4809.633 \pm 0.002$ & 207.916 & $2.2 \pm 0.2$ & 12 & $4+6$ \\
$5009.696 \pm 0.002$ & 199.613 & $0.7 \pm 0.2$ & 4 & $2 \mathrm{f} 4$ \\
$5155.187 \pm 0.002$ & 193.979 & $2.2 \pm 0.2$ & 8 & $1+1+2$ \\
$5361.052 \pm 0.002$ & 186.531 & $1.2 \pm 0.2$ & 6 & $3+10$ \\
$5408.698 \pm 0.002$ & 184.887 & $1.3 \pm 0.2$ & 6 & $3+3+1$ \\
$5913.800 \pm 0.002$ & 169.096 & $1.4 \pm 0.2$ & 7 & $1+4+5$ \\
\hline & & & &
\end{tabular}

Note. ${ }^{\text {a }}$ Combination including parent with amplitude modulation.

squared residuals, $\operatorname{Res}_{\mathrm{rms}}$, between the predicted and observed amplitudes. In practice, we run the code 1000 times and select 


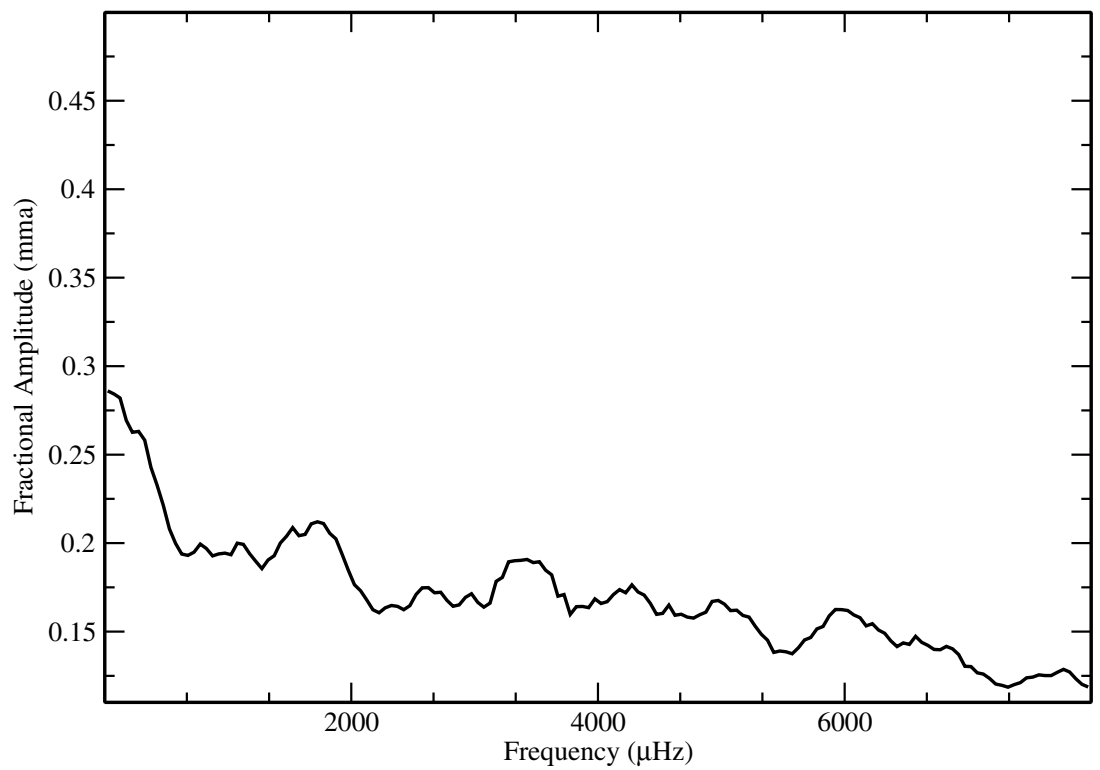

Figure 6. Mean noise as a function of frequency for XCOV26. The data set was prewhitened by the 62 largest amplitude frequencies. Our noise is frequency dependent, but is near $0.2 \mathrm{mma}$. This is a conservative estimate, as we have probably not prewhitened by all the combination frequencies.

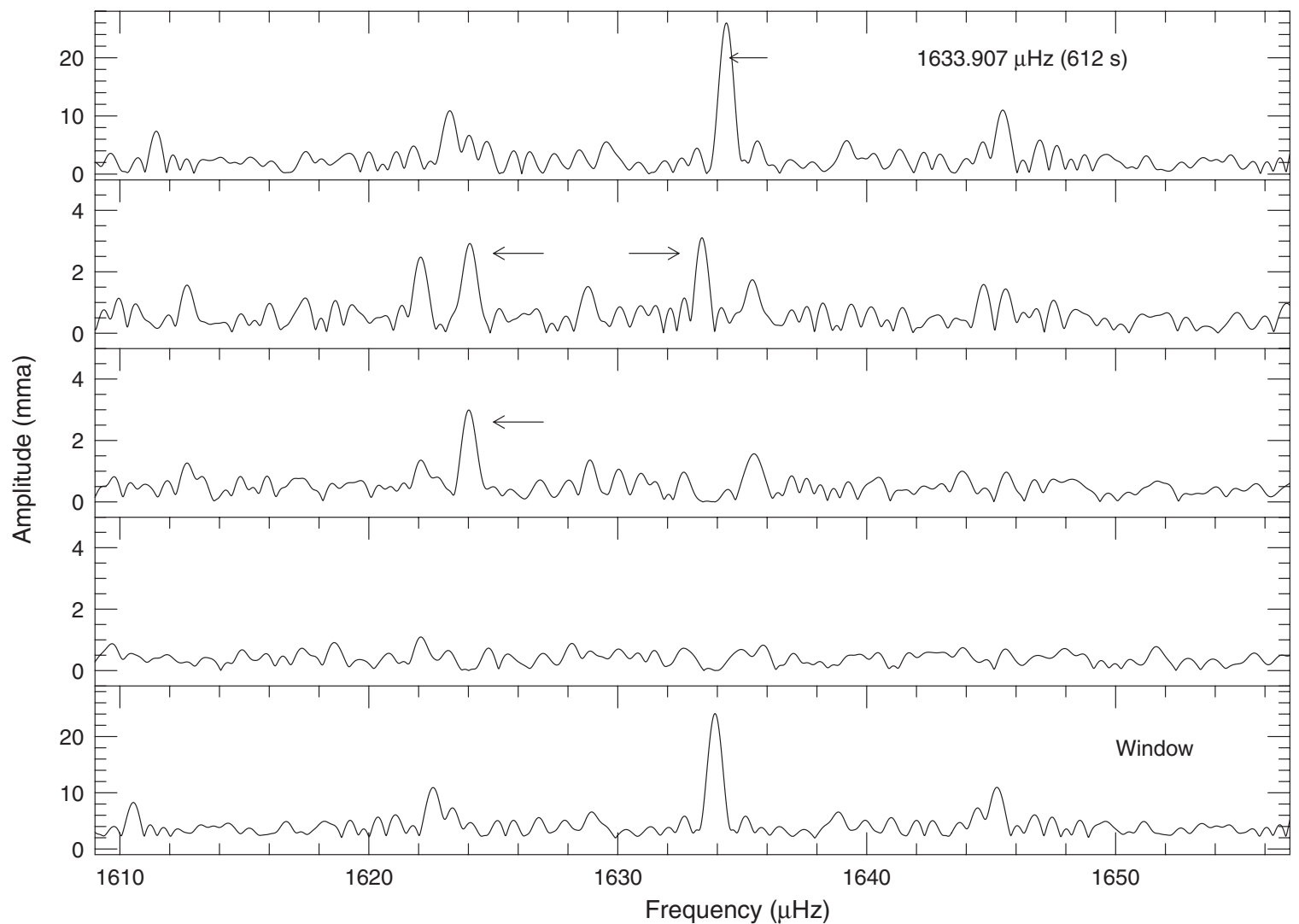

Figure 7. Prewhitening of the dominant $1633.907 \mu \mathrm{Hz}$ mode in the $2008 \mathrm{FT}$. We begin with the removal of the largest amplitude resolved peak (top panel), a careful comparison of the residuals in the next panel with the spectral window (last panel), and the subsequent removal of additional frequencies. The fourth panel shows the residuals after simultaneously removing $1633.907,16233.450$, and $1624.017 \mu \mathrm{Hz}$. The $1633.450 \mu \mathrm{Hz}$ component (right arrow in the second panel) is the result of amplitude changes.

the solutions having Res $_{\text {rms }}$ below a limiting value. This process produces a sample of best-fit solutions whose distribution provides information on the range of values allowed for these parameters.

To test that this approach recovers known input, we used the nonlinear light curve fitting code of Montgomery et al. (2010b) to generate a synthetic light curve based on EC14012-1446's parent frequencies. The nonlinear light curve fitting code is discussed in more detail in Section 5. For our purposes in this test, the importance of using synthetic light curves generated by this code rather than a simple simulation employing multiple sine functions is that the synthetic light curve will include 


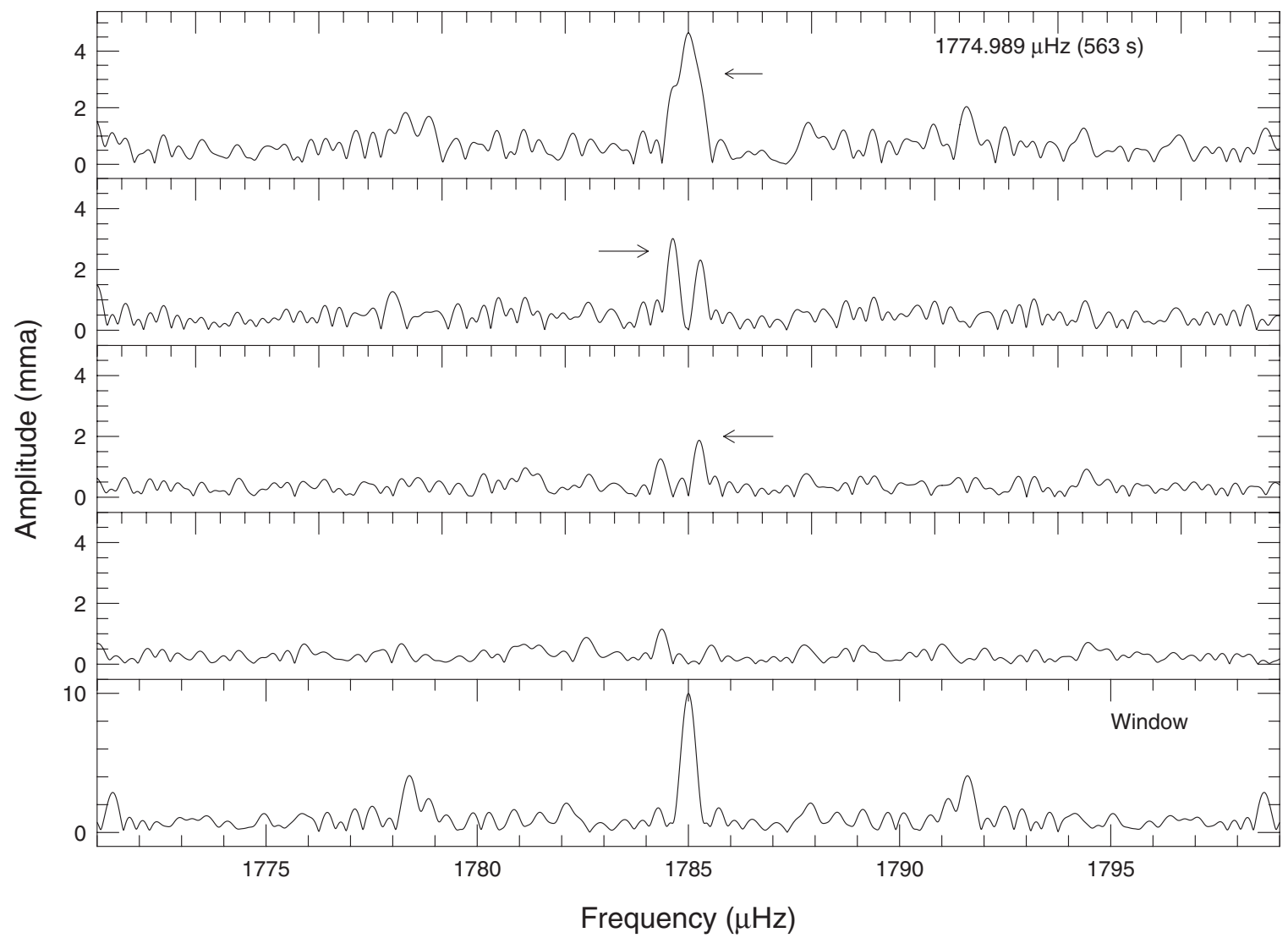

Figure 8. $1775 \mu \mathrm{Hz}(563 \mathrm{~s})$ mode in the 2008 FT. This power is unresolved and requires at least four closely space frequencies for prewhitening, with frequency differences very similar to the inverse of the run length. This is a clear signature of amplitude and/or frequency instability.

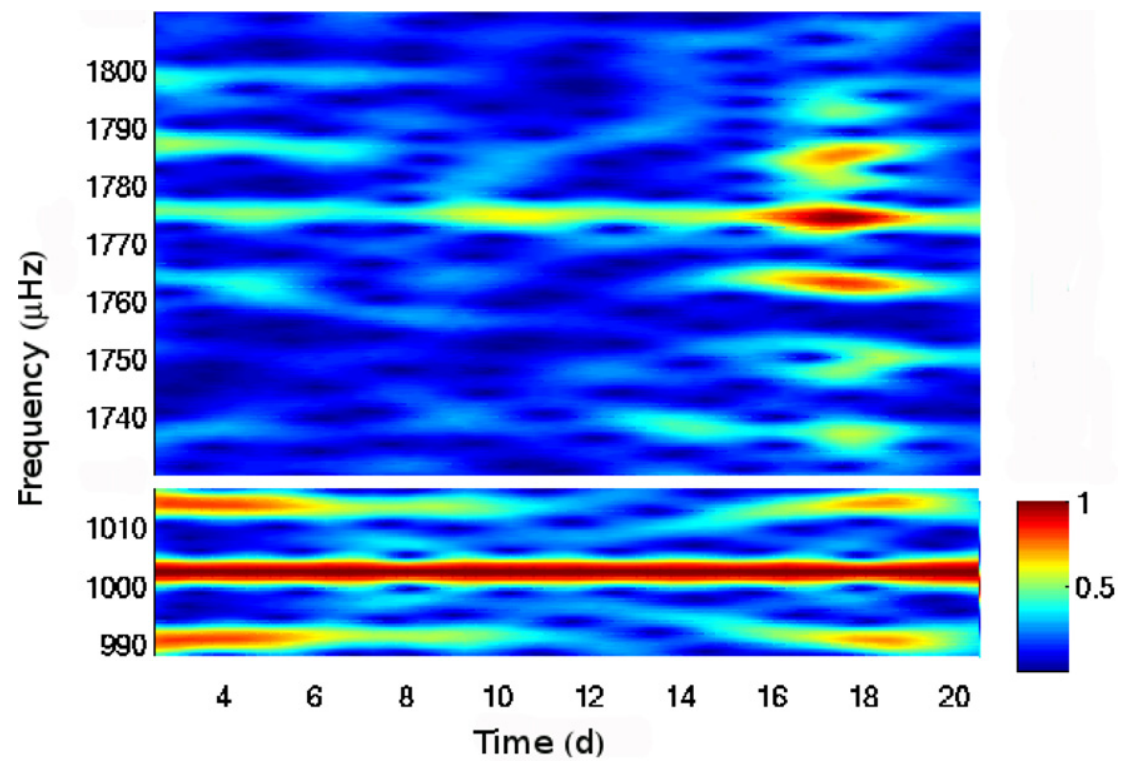

Figure 9. Spectrogram of the $1775 \mu \mathrm{Hz}$ region in the $2008 \mathrm{FT}$. This region of power exhibits remarkable amplitude changes on a timescale of days. The bottom panel gives the time-dependent window. The spectrogram amplitudes (colors) have been normalized to a value of 1 .

(A color version of this figure is available in the online journal.)

combination frequencies produced via nonlinear effects due to convection (Montgomery et al. 2010b). Our purpose is to recover the input frequency identifications using these combination frequencies. We assigned reasonable $(l, m)$ values to the input frequencies, chose values for $\theta$, the time-averaged convective response time $\tau_{0}$, and $A_{0}$, where $A_{\mathrm{o}}$ describes the response of the stellar material to the pulsations and includes a bolometric correction factor (Wu 2001), and added noise. Using the method outlined in the previous paragraph, we successfully recovered $(l, m)$ for all large-amplitude input parent frequencies, while experiencing some disagreement with low-amplitude parents. This is because low-amplitude modes have even lower amplitude 

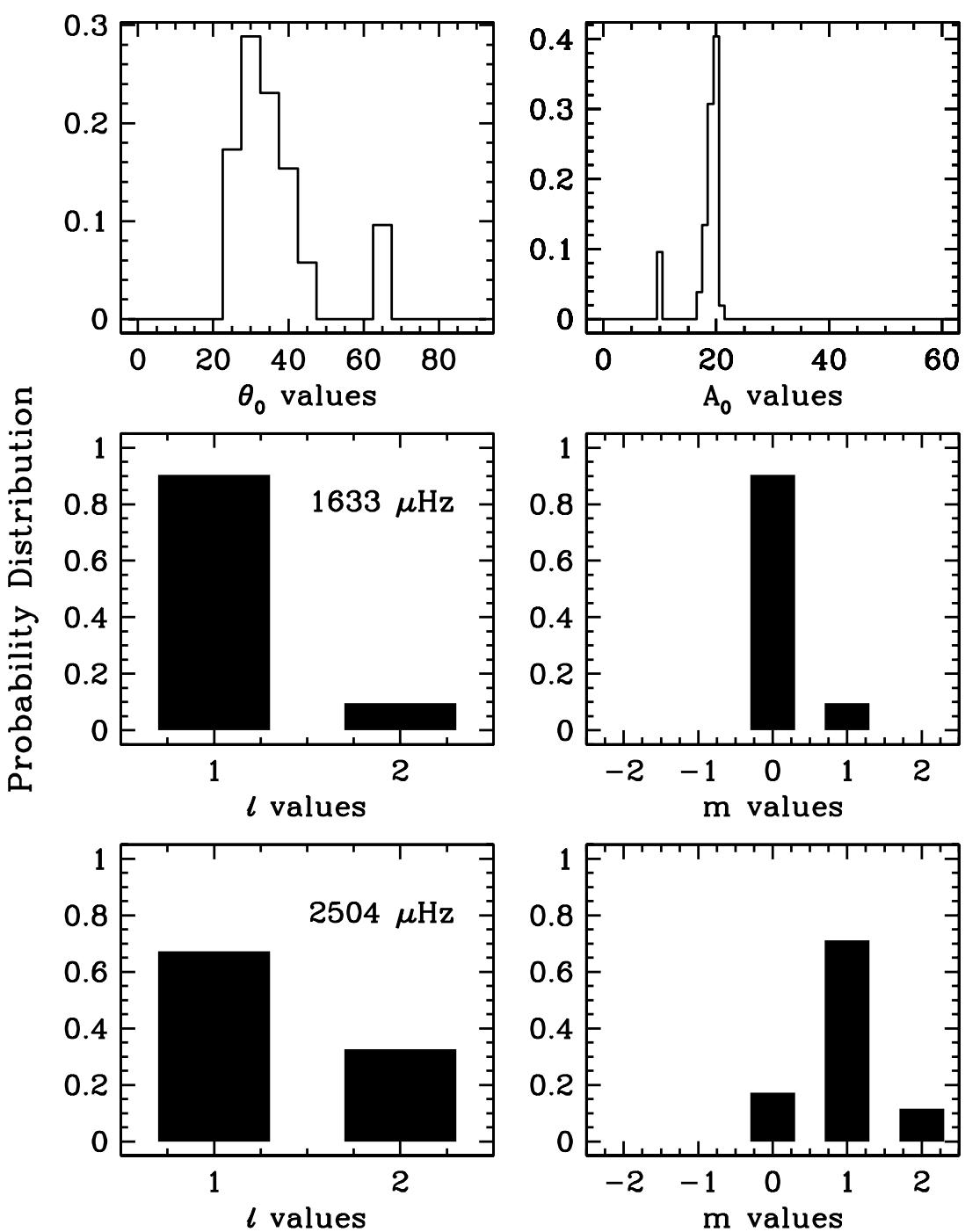

Figure 10. Probability distribution (from 0 to 1 ) of $l, m, \theta$, and $A_{\mathrm{o}}$ for the $1633.907 \mu \mathrm{Hz}(612 \mathrm{~s}$ ) and the $2504.897 \mu \mathrm{Hz}$ (399 s) variations. The solutions are from individual runs with Res $\mathrm{rms}_{\mathrm{rm}}<0.38 \mathrm{mmi}$. The top panel gives the probability distributions of $\theta$ and $A_{\mathrm{o}}$ found for all frequencies, with preferred values of $\theta \sim 32^{\circ}$ and $A_{\mathrm{o}} \sim 20$, respectively. The middle and lower panels give the $l$ and $m$ identification distributions for the $1633.907 \mu \mathrm{Hz}(612 \mathrm{~s})$ and $2504.897 \mu \mathrm{Hz}(399 \mathrm{~s})$ modes. The amplitudes of EC14012-1446's observed combination frequencies argue that $1633.907 \mu \mathrm{Hz}$ is $l=1, m=0$, while $2504.897 \mu \mathrm{Hz}$ is $l=1, m=1$.

combination frequencies, which are difficult to detect and so are not as numerous. Since our method treats both the highamplitude parent modes and the low-amplitude combination frequencies equally in the fits, this has the effect of deemphasizing the importance of low-amplitude combination frequencies.

Figure 10 shows the resulting probability distribution of $l, m, \theta$, and $A_{\mathrm{o}}$ values for fits having $\operatorname{Res}_{\mathrm{rms}}<0.38 \mathrm{mmi}$, for EC14012-1446's $1633.907 \mu \mathrm{Hz}(612 \mathrm{~s})$ and $2504.897 \mu \mathrm{Hz}$ (399 s) variations. For $1633.907 \mu \mathrm{Hz}(612 \mathrm{~s})$, the analysis of combination amplitudes strongly argues that the variations are best represented as spherical degree $l=1$ and azimuthal index $m=0$, with an inclination angle of $30^{\circ} \pm 10^{\circ}$, and a value of $A_{\mathrm{o}}=20 \pm 2$. We find nearly identical results for $1887.404 \mu \mathrm{Hz}$ (529 s) and $1548.146 \mu \mathrm{Hz}$ (646 s). For $2504.897 \mu \mathrm{Hz}(399 \mathrm{~s})$, we find the same distributions for $\theta$ and $A_{\mathrm{o}}$, but in this case $(l, m)=(1,1)$ is strongly preferred. As we found previously, the statistical significance of $(l, m)$ identifications determined by combination amplitudes is amplitude dependent and we do not find unambiguous identifications for the lower amplitude frequencies. The complete list of $(l, m)$ identifications derived from the combination amplitudes are given in Table 4.
Our analysis of EC14012-1446's combination amplitudes indicates that nine of the dominant independent frequencies are consistent with spherical degree $l=1$; in Table 4 we list the most likely $(l, m)$ identifications for these modes. We would like to constrain the identifications of the remaining independent frequencies to improve our chances of success with nonlinear convective light curve fitting. Using the combination analysis as a basis, we now turn to asteroseismology to provide constraints on the identifications of the remaining frequencies.

\subsection{Asteroseismology}

\subsubsection{Period Spacing}

As mentioned in the introduction, we look for two clues to indicate $(l, m)$ identifications of pulsations in white dwarfs. The first is the expectation that $g$-mode pulsations of a given $l$ corresponding to successive radial overtones $k$ will be approximately equally spaced in period, provided $k$ is large enough (Unno et al. 1989). For the simple example of a homogeneous star, we find

$$
P_{k l}=k \Delta \Pi[l(l+1)]^{-1 / 2}+C,
$$


Table 4

Frequency $(l, m)$ Identifications

\begin{tabular}{|c|c|c|c|c|}
\hline $\begin{array}{l}\text { Frequency } \\
(\mu \mathrm{Hz})\end{array}$ & $\begin{array}{l}\text { Period } \\
\text { (s) }\end{array}$ & $\begin{array}{l}\text { Combination } \\
\qquad(l, m)\end{array}$ & $\begin{array}{c}\text { Asteroseismology } \\
(l, m)\end{array}$ & $\begin{array}{l}\text { Final Nonlinear Fit } \\
\qquad(l, m)\end{array}$ \\
\hline $935.3799 \pm 0.002$ & 1069.085 & & $(1$, any $)$ & $(2,-1)$ \\
\hline $1021.139 \pm 0.002$ & 979.299 & & $(1$, any $)$ & $(2,2)$ \\
\hline $1104.252 \pm 0.001$ & 905.591 & & (1, any) & $(2,1)$ \\
\hline $1155.925 \pm 0.002$ & 865.108 & & (1, any) & $(2,2)$ \\
\hline $1241.403 \pm 0.002$ & 805.540 & & $(1$, any $)$ & $(2,2)$ \\
\hline $1418.369 \pm 0.002$ & 705.035 & & (2, any) & $(2,1)$ \\
\hline $1521.575 \pm 0.002$ & 657.214 & & $(1,1)$ & $1,1)$ \\
\hline $1548.146 \pm 0.001$ & 645.933 & $(1,0)$ & (1, any) & $(1,0)$ \\
\hline $1624.015 \pm 0.003$ & 615.758 & $(1,1)$ & $(1,1)$ & $(1,1)$ \\
\hline $1633.907 \pm 0.001$ & 612.030 & $(1,0)$ & $(1,0 / 1)$ & $(1,1)$ \\
\hline $1774.989 \pm 0.100$ & 563.384 & $(1,-1)$ & (1, any) & $(1,1)$ \\
\hline $1860.248 \pm 0.400$ & 537.563 & & (2, any) & $(2,0)$ \\
\hline $1883.555 \pm 0.003$ & 530.911 & & $(1,1)$ & $(1,1)$ \\
\hline $1887.404 \pm 0.001$ & 529.828 & $(1,0)$ & $(1,0)$ & $(1,0)$ \\
\hline $1891.142 \pm 0.002$ & 528.781 & $(1,-1)$ & $(1,-1)$ & $(1,-1)$ \\
\hline $2304.745 \pm 0.001$ & 433.887 & $(1,-1)$ & (2, any) & $(1,0)$ \\
\hline $2504.897 \pm 0.001$ & 399.218 & $(1,1)$ & $(1,1)$ & $(1,0)$ \\
\hline $2508.060 \pm 0.002$ & 398.715 & $(1 / 2,1 / 0)$ & $(1,0)$ & $(1,-1)$ \\
\hline $2856.155 \pm 0.002$ & 350.121 & $(2,0 / 1)$ & (2, any) & $(2,0)$ \\
\hline
\end{tabular}

where $\Delta \Pi$ is a uniform period spacing, $P_{k l}$ is the period for a given mode $(k, l)$, and $C$ is a constant (Tassoul et al. 1990). The mean period spacing $\Delta \Pi$ for a series of modes of a given $l$ and consecutive $k$ is an important asteroseismic measure of stellar mass and effective temperature that is mostly independent of internal composition (Unno et al. 1989).

A white dwarf has a host of available pulsation frequencies. For reasons that are not understood, in most cases only a subset are observed at any given time (Córsico et al. 2002). This is true for EC14012-1446. The 19 independent frequencies detected during XCOV28 show no obvious evidence of equal period spacing so we are lacking consecutive radial overtones for any $l$ value. A good strategy to identify the complete set of available modes is to combine results from multiple seasons of observations. Handler et al. (2008) present an analysis of EC14012-1446 observations spanning 2004-2007, during which the star exhibited different subsets of excited modes. Figure 11 shows a schematic representation of excited modes for the combined observations spanning 2004-2008. Figure 12 focuses on the obvious groupings between 800 and $500 \mathrm{~s}$ $(1250$ and $2000 \mu \mathrm{Hz})$. Since we are searching for equal period spacings, we present these figures using period as the $x$-axis. The large groupings at $768 \mathrm{~s}(1302 \mu \mathrm{Hz}), 721 \mathrm{~s}(1387 \mu \mathrm{Hz}), 682 \mathrm{~s}$ $(1466 \mu \mathrm{Hz})$, and $612 \mathrm{~s}(1633 \mu \mathrm{Hz})$ have a decreasing observed width with shorter period. This decrease translates into an equal width in frequency space of $\approx 24 \mu \mathrm{Hz}$.

We calculated a simple average period for each group, which is given in Table 5. A statistical test for the presence of uniform period spacing is provided by the Kolmogorov-Smirnov (K-S) test (Winget et al. 1991). The K-S test calculates the probability that an input list is randomly distributed. Any statistically nonrandom period spacing will therefore appear as a minimum value in the output. In our case, we use the test to determine the probability that the list of average periods in Table 5 is from a uniform distribution for a given period spacing $\Delta \Pi$ (Kawaler 1988). Figure 13 shows the results, finding a period spacing of $\approx 41 \mathrm{~s}$, consistent with the expectations for $l=1$ (Bischoff-Kim $\&$ Metcalfe 2012). We find no significant period spacings at $\approx 23 \mathrm{~s}$ as predicted for $l=2$ modes.
Table 5

Table of Average Frequencies (2004-2008)

\begin{tabular}{lccc}
\hline \hline $\begin{array}{l}\text { Period } \\
(\mathrm{s})\end{array}$ & $\begin{array}{c}\text { Frequency } \\
(\mu \mathrm{Hz})\end{array}$ & No. of peaks & Radial Overtone \\
\hline 2856.164 & 350.120 & 1 & $k_{\mathrm{o}}-6$ \\
2738.076 & 365.220 & 1 & $k_{\mathrm{o}}-5$ \\
2505.405 & 399.137 & 6 & $k_{\mathrm{o}}-2$ \\
2304.854 & 433.865 & 2 & \\
1887.505 & 529.800 & 10 & $k_{\mathrm{o}}-1$ \\
1860.431 & 537.510 & 2 & $k_{\mathrm{o}}$ \\
1774.954 & 563.395 & 2 & $k_{\mathrm{o}}+1$ \\
1633.597 & 612.146 & 16 & \\
1548.155 & 645.930 & 2 & $k_{\mathrm{o}}+3$ \\
1463.668 & 683.215 & 8 & $k_{\mathrm{o}}+4$ \\
1418.353 & 705.040 & 1 & $k_{\mathrm{o}}+5$ \\
1384.903 & 722.072 & 14 & $k_{\mathrm{o}}+6$ \\
1332.851 & 750.249 & 1 & $k_{\mathrm{o}}+7$ \\
1298.863 & 769.904 & 6 & $k_{\mathrm{o}}+9$ \\
1219.836 & 819.782 & 2 & $k_{\mathrm{o}}+11$ \\
1163.163 & 859.710 & 2 & \\
1136.538 & 879.865 & 4 & \\
1104.240 & 905.600 & 1 & \\
1035.443 & 965.770 & 2 & 3 \\
964.748 & 1036.540 & 1 & \\
935.375 & 1069.090 & 1 & \\
821.254 & 1217.650 & & \\
\hline
\end{tabular}

Notes. This table gives the simple average frequencies for the groupings in Figures 11 and 12. The column "No. of peaks" gives the number of frequencies contained within each group. The radial overtone $k$ is impossible to determine observationally, so we give values relative to $1633 \mu \mathrm{Hz}$, defined to have $k_{\mathrm{o}}$. The relative $k$ values denote a series of nearly consecutive modes of spherical index $l=1$ with a period spacing of $\approx 41 \mathrm{~s}$.

Using $1633.907 \mu \mathrm{Hz}$ as our reference point, we assigned relative radial overtone values to the frequencies listed in Table 5. We plot the expected locations of consecutive radial overtones for $l=1$ in Figures 11 and 12. These points in the bottom of both figures are given with bars representing the $24 \mu \mathrm{Hz}$ "grouping" width. For periods above $900 \mathrm{~s}$, the grouping 


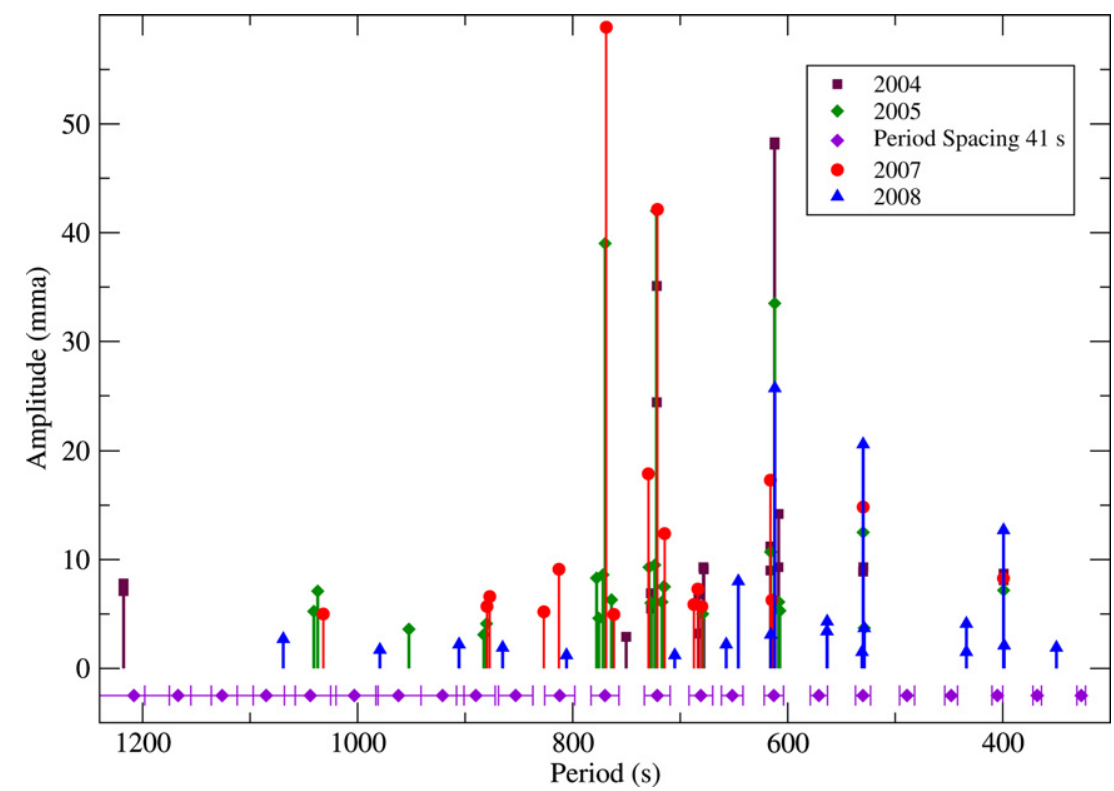

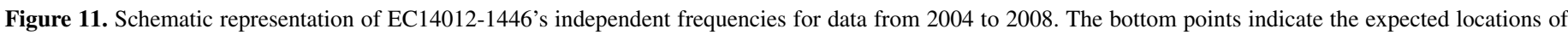

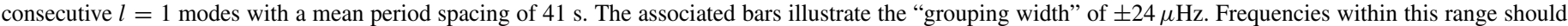
belong to the given $k$ multiplet. Note that the $x$-axis is period, not frequency.

(A color version of this figure is available in the online journal.)

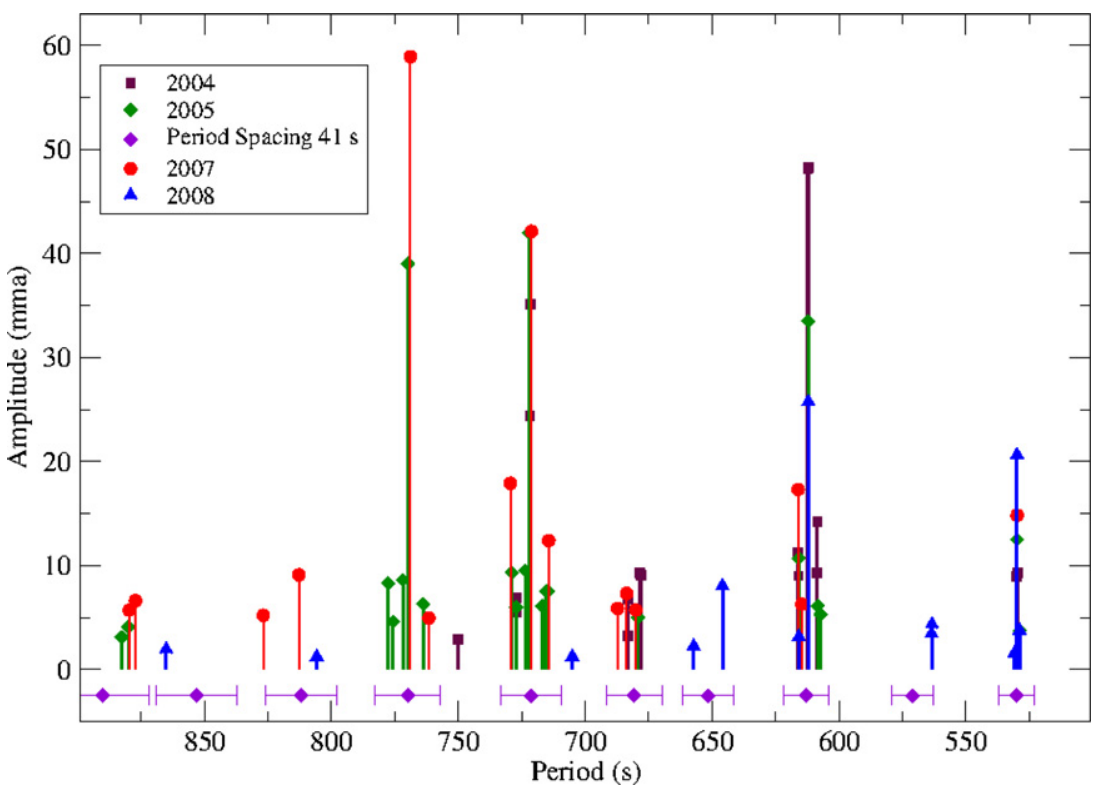

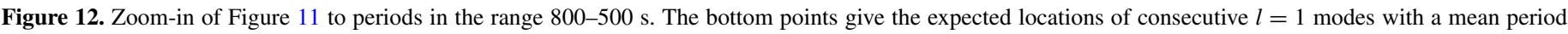

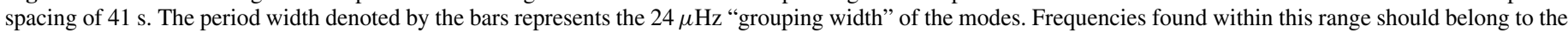
given $k$ multiplet. Please note that the $x$-axis is period, not frequency.

(A color version of this figure is available in the online journal.)

widths for consecutive radial overtones overlap, illustrating the difficulty of assigning relative $k$ values to modes with periods longer than this value. Nonetheless, we find that most of EC14012-1446's pulsation frequencies can be identified as $l=1$.

Unlike our simple example of a homogeneous star, white dwarfs are compositionally stratified, so the individual period spacings will not be uniform. We can retrieve detailed information about interior structure from the distribution of excited pulsation frequencies. A long-standing problem with the asteroseismology of DA pulsating white dwarfs is the lack of objects with rich pulsation spectra (Bischoff-Kim \& Metcalfe 2012). In this respect, EC14012-1446 immediately reveals its poten- tial. Using our calculated average periods from Table 5, we show how individual period spacings $\Delta P$ differ as a function of period (and relative $k$ value) in Figure 14. We use "forward differencing," where $\Delta P$ is defined as $\Delta P=P_{k}-P_{k+1}$. The filled points represent the periods between $\approx 900$ and 500 s that we are certain are consecutive radial overtones of $l=1$. The open circles represent those periods above $\approx 900 \mathrm{~s}$ with ambiguous $k$ identifications. The roughly cyclic behavior in Figure 14 is a sign of mode trapping. Mode trapping occurs in compositionally stratified stars when there is a resonance between a pulsation frequency and a surface layer. In theoretical models, a resonance occurs when a radial node coincides with a transition layer. In a DA white dwarf, transition zones occur at the 


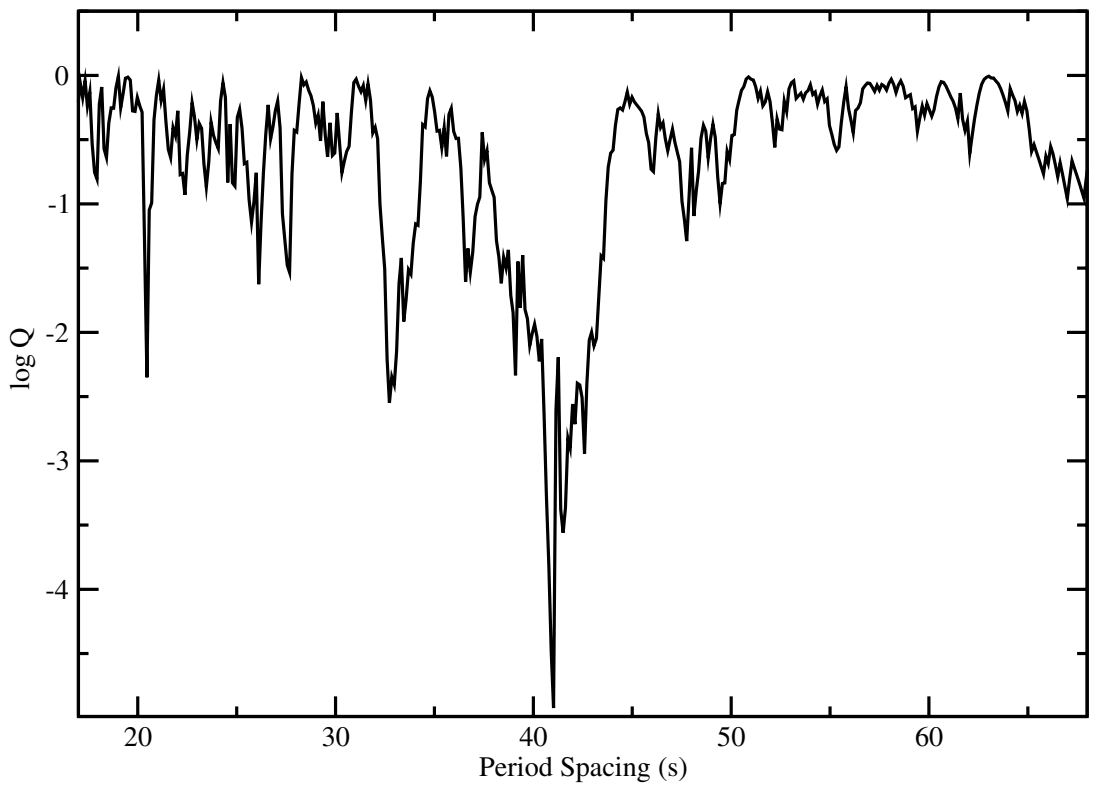

Figure 13. K-S test applied to the average periods in Table 5. A period spacing is defined as significant with a confidence level of $(1-Q) \times 100 \%$. The results reveal an average period spacing of $\approx 41 \mathrm{~s}$ with a confidence level of $99.99 \%$.

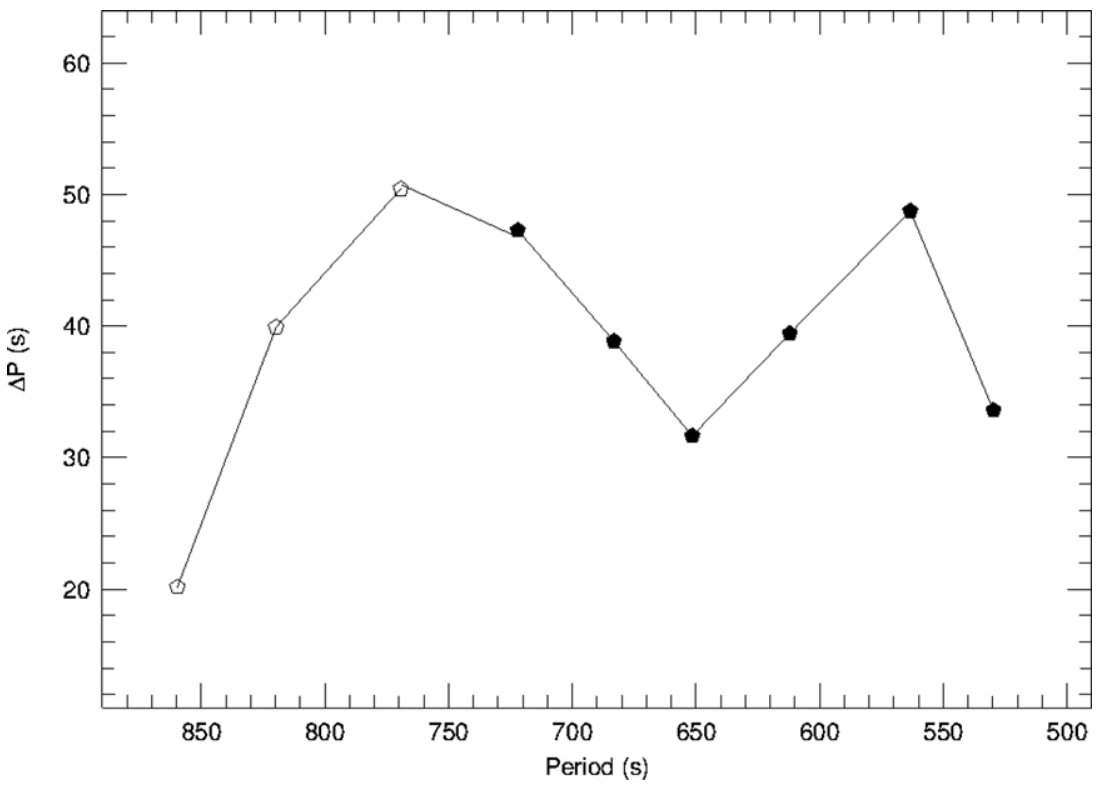

Figure 14. $\Delta P$ vs. $P$ for EC14012-1446, using the average periods from Table 5. We use forward differencing $\left(\Delta P=P_{k}-P_{k+1}\right)$, where the radial overtone $k$ increases to the left. The solid points represent those frequencies with $l=1$ identifications. The open points represent those where our identification is uncertain.

boundary of the hydrogen and helium layers, the helium layer and the carbon/oxygen core, and at points in a possibly chemically stratified core (Montgomery 2005b). The trapping cycle (the number of frequencies from minimum to minimum) is most sensitive to the location of the trapping layer, e.g., the base of the surface hydrogen layer. In addition, the trapping amplitude (the depth of the minima) is sensitive to the density gradient in the composition transition zone. In general, a larger gradient produces a larger trapping amplitude. Work is underway to determine EC14012-1446's detailed mass and internal structure (Bischoff-Kim \& Metcalfe 2012).

\subsubsection{Multiplet Structure}

A second clue to constrain $(l, m)$ identifications of pulsations in white dwarfs is the presence of rotationally split multiplets. The multiplet components have the same $(k, l)$ and are further described by the azimuthal index $m$, which takes integer values between $-l$ and $l$. To first order, the frequency difference relative to the $m=0$ component of the multiplet is given by $\delta v_{k l m}=-m \Omega\left(1-C_{k l}\right)$, where $\Omega$ is the rotation period and $C_{k l}$ is a coefficient that depends on the pulsation eigenfunctions evaluated in the nonrotating case. In the high- $k$ asymptotic limit for $g$-modes, $C_{k l} \sim 1 / \ell(\ell+1)$, although models predict it to vary by $\approx 10 \%$ over the range of observed periods in EC14012. We adopt the convention that a positive value of $m$ represents a prograde mode; retrograde modes are denoted by negative $m$ values (Winget et al. 1994). An additional important diagnostic is given by the ratio of splittings for $l=1$ and $l=2$, where we expect $\delta v_{\ell=1} / \delta v_{\ell=2} \simeq 0.6$ for $g$-modes (Winget et al. 1991).

For EC14012-1446 during XCOV26, we find one triplet $(1887 \mu \mathrm{Hz})$ and two doublets (1633 and $2504 \mu \mathrm{Hz})$ among our list of 19 frequencies (Table 2). We begin by assuming that the 
$1887 \mu \mathrm{Hz}$ triplet is a rotationally split multiplet. Our combination analysis argues that the central component $(1887.404 \mu \mathrm{Hz}$, $529.828 \mathrm{~s})$ is $(1,0)$ and the $1891.142 \mu \mathrm{Hz}(528.781 \mathrm{~s})$ component is $(1,-1)$. The $1883.555 \mu \mathrm{Hz}$ (530.911 s) component is low amplitude and is not identified as a parent of any detected combination frequencies. We cannot constrain its $(l, m)$ identification via that method. Given $(l, m)$ identifications for the first two modes, the $1883.555 \mu \mathrm{Hz}$ component should be $(1,1)$. The average multiplet splitting of $3.8 \mu \mathrm{Hz}$ implies a rotation period as sampled by these modes of $1.53 \pm 0.01$ days. This is a perfectly reasonable result, as spectroscopic studies of white dwarfs reveal upper limits of $v \sin i=10 \mathrm{~km} \mathrm{~s}^{-2}$, consistent with rotation periods of order days or longer (Berger et al. 2005).

In the limit of uniform slow rotation, we expect additional $l=1$ multiplets to exhibit similar splittings and $l=2$ modes to have splittings near $6.3 \mu \mathrm{Hz}$ (Winget et al. 1991). Recalling that every $m$ component is not necessarily excited, we turn to the doublet at $1633 \mu \mathrm{Hz}(612 \mathrm{~s})$. Period spacing argues that this doublet is $l=1$, and our combination analysis indicates that the $1633.907 \mu \mathrm{Hz}(612.03 \mathrm{~s})$ mode is $(1,0)$. The $1624.015 \mu \mathrm{Hz}$ $(615.758 \mathrm{~s})$ component is not found as a parent of any detected combination frequencies. If $1633.907 \mu \mathrm{Hz}$ is indeed the central component of an $l=1$ triplet, then $1624.015 \mu \mathrm{Hz}$ is $m=1$ and we do not detect the $m=-1$ component. However, the splitting of $9.89 \mu \mathrm{Hz}$ differs significantly from the expected value of $\approx 3.8 \mu \mathrm{Hz}$ found for the $1887 \mu \mathrm{Hz}$ triplet. A second possibility is that the combination analysis is incorrect and we are missing the multiplet's central component. In this case, the $1633.907 \mu \mathrm{Hz}$ mode is $(1,-1)$ and the $1624.015 \mu \mathrm{Hz}$ is $(1,1)$, resulting in an average splitting of $4.95 \mu \mathrm{Hz}(9.89 / 2)$. Moving to the $2504 \mu \mathrm{Hz}$ (399 s) doublet, period spacing again argues that this multiplet is $l=1$. Our combination analysis indicates that the $2504.987 \mu \mathrm{Hz}(399.204 \mathrm{~s})$ component is $(1,1)$. Again, the $2508.060 \mu \mathrm{Hz}$ (398.715 s) component is not identified as a parent of any detected combination frequency. The frequency splitting between the two components is $3.16 \mu \mathrm{Hz}$ and by comparison with the splitting of the $1887 \mu \mathrm{Hz}$ triplet, we can argue that these modes have consecutive $m$ values, identifying the $2508.060 \mu \mathrm{Hz}$ component as $(1,0)$.

Multiplet splittings may also be used to eliminate the possibility that EC14012-1446's frequencies represent a mixture of $l=1$ and $l=2$ modes. Assuming the $1887 \mu \mathrm{Hz}$ triplet is $l=1$, we do not find evidence for $l=2$ splittings of $\delta v_{l=2}=(3.8 / 0.6)=6.3 \mu \mathrm{Hz}$ as predicted in the limit of slow, uniform rotation. Although both the period spacing and the combination analysis argue against it, to play devil's advocate we consider the possibility that the $1887 \mu \mathrm{Hz}$ triplet is actually an $l=2$ quintuplet, since all $m$ components are not necessarily excited to observable levels. In this case, then the expected $l=1$ multiplet splitting is $\delta v_{l=1}=3.8 \times 0.6=2.3 \mu \mathrm{Hz}$. We find no examples of multiplet splittings near this value. Finally, we must consider that $\delta v_{3.16} / \delta v_{4.95}=0.64$. Based on multiplet structure alone, we could argue that the $1633.907 \mu \mathrm{Hz}$ frequency is $l=2$, and $2504.897 \mu \mathrm{Hz}$ is $l=1$, but this is not supported by either the period spacings or the combination analysis and leaves no clear explanation for the $1887 \mu \mathrm{Hz}$ triplet.

Clearly, the multiplets found in EC14012-1446's XCOV26 data set are not well explained based on the simple model of rotational splitting. Multiplet structure should be determined by the star's rotation rate and structure. We expect this to remain unchanged over time. The classical example is PG1159-035, which exhibits triplets and quintuplets corresponding to $l=1$ and $l=2$ (Winget et al. 1991). However, complex multiplets are not unusual for white dwarf pulsators. In some instances, the assumption of rigid rotation is clearly violated (Córsico et al. 2011). In cooler pulsators with moderate amplitudes and well-developed convection zones, observed multiplet structure can exhibit complicated behavior. For example, Provencal et al. (2009) show changes in the DBV GD358's multiplet structure that cannot be explained with simple rotational splitting. For this same star, Winget et al. (1994) show a dependence of multiplet splittings that is not explained by expected variations of $C_{k l}$. Processes that may play a role in multiplet structure of cooler pulsators include changing weak magnetic fields similar to the solar cycle, oblique pulsation, and differential rotation. Our XCOV26 data on EC14012-1446 provide us with a single snapshot of this star's multiplet structure. We need more observations to understand their behavior.

We turn again to the combined observations from 2004 to 2008. The four large groupings at $768 \mathrm{~s}(1302 \mu \mathrm{Hz}), 721 \mathrm{~s}$ $(1387 \mu \mathrm{Hz}), 682 \mathrm{~s}(1466 \mu \mathrm{Hz})$, and $612 \mathrm{~s}(1633 \mu \mathrm{Hz})$ in Figure 12 all show indications of multiplet structure within their groups. We extracted an average multiplet for each of the four large groupings in Figure 12 by calculating a simple average frequency value for the $m=1, m=0$, and $m=+1$ components. The results are presented in Figure 15. In each case, the prograde $(m=+1)$ mode splitting is larger than the $m=-1$ mode, and both the splittings and the asymmetries increase with increasing radial node $k$.

The qualitative behavior of the low- $k$ and high- $k$ modes can be explained in terms of a very general model. $g$-modes are standing waves of buoyancy in a spherical cavity and can be thought of as superpositions of traveling waves bouncing back and forth between an inner and outer turning point. In general, low- $k$ (shorter period) modes have deeper outer turning points while high- $k$ (longer period) modes have turning points closer to the stellar surface, meaning that these modes sample the outer regions of the star more than do low- $k$ modes. Figure 15 shows that the unknown process affecting EC14012-1446's multiplet structure acts more dramatically on the high- $k$ modes, arguing that the structural perturbation must be in the outer layers. A surface magnetic field and/or the convection zone are obvious candidates. In addition, we should consider both radial and latitudinal differential rotation. Work is underway to improve our understanding of multiplet structure in pulsating white dwarfs (J. Dalessio 2012, private communication).

Asteroseismology of EC14012-1446's distribution of excited frequencies shows that the observed pulsations are dominated by a series of $l=1$ modes. If $l=2$ modes are present, the modes are low amplitude and will not greatly affect the nonlinear light curve fitting process. For the 1887, 1663, and $2504 \mu \mathrm{Hz}$ multiplets, we are able to constrain the $m$ identifications as well. The combination of these results with the combination amplitude analysis (Table 4) gives us a strong foundation on which to proceed to nonlinear light curve fitting.

\section{NONLINEAR ANALYSIS}

Montgomery (2005a) and Montgomery et al. (2010b) give a detailed description of fitting observed non-sinusoidal light curves of white dwarfs to extract the time-averaged thermal response timescale of the convection zone. To summarize, the aspect of the convection zone sampled by the pulsations is the thermal response timescale, $\tau_{\mathrm{c}}$, which is directly related to the convection zone's mass and depth, and therefore its 


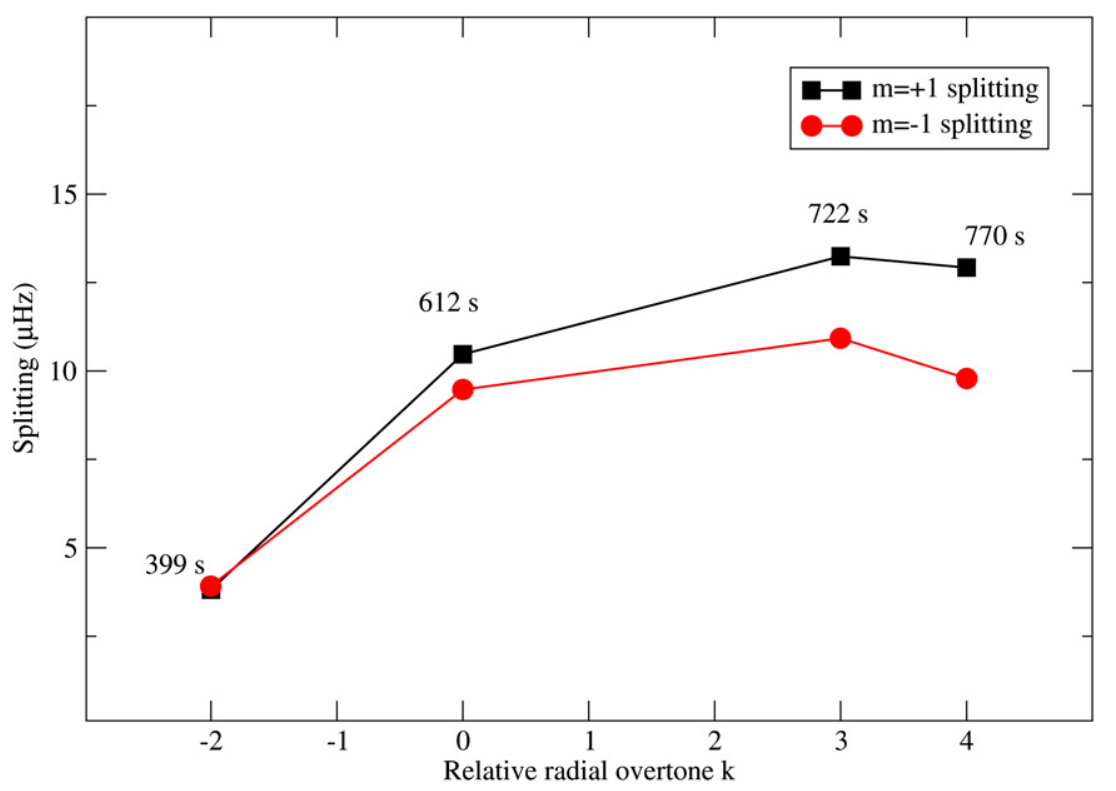

Figure 15. Average multiplet structure associated with the large groupings at $770 \mathrm{~s}(1302 \mu \mathrm{Hz}), 722 \mathrm{~s}(1387 \mu \mathrm{Hz}), 612 \mathrm{~s}(1633 \mu \mathrm{Hz})$, and $399 \mathrm{~s}(1887 \mu \mathrm{Hz})$. The $m=+1$ (prograde) splitting is always larger than the $m=-1$ splitting, and the asymmetry increases with increasing $k$.

(A color version of this figure is available in the online journal.)

heat capacity. A DAV such as EC14012-1446 will experience local temperature excursions of $\pm 250 \mathrm{~K}$. In response, the local mass and depth of the convection zone will vary throughout a pulsation cycle.

MLT predicts that $\tau_{\mathrm{c}}$ should scale as

$$
\tau_{c} \approx \tau_{0}\left(T_{\mathrm{eff}} / T_{\mathrm{eff}, 0}\right)^{-N},
$$

where $T_{\text {eff }}$ is the instantaneous effective temperature, $T_{\text {eff, } 0}$ is the equilibrium $T_{\text {eff }}, \tau_{0}$ is the time-averaged convective timescale, and $N$ describes the sensitivity of $\tau_{c}$ to changes in $T_{\text {eff }}$. For DAs, MLT predicts $N \approx 90$ (Montgomery 2005a; Wu 2001; Brickhill 1992). The convection zone's mass, depth, and heat capacity are therefore extraordinarily temperature sensitive, and can vary by a factor of $\approx 10$ throughout a pulsation cycle. This modulation of depth and heat capacity is the source of the large nonlinearities in white dwarf light curves. We focus on these observed nonlinearities to determine the time-averaged convective timescale $\tau_{0}$, the temperature sensitivity parameter $N$, and the inclination of the pulsation axis to the line of sight $\theta$.

Our analysis follows the approach of Montgomery et al. (2010b). EC14012-1446 is a multiperiodic pulsator, and because of nonlinear effects, a pulse shape obtained by folding its light curve at a period of interest is not equivalent to a pulse shape obtained in the absence of additional frequencies (Montgomery 2007). Therefore, we use the accurate frequencies and $(l, m)$ identifications obtained for the 2008 WET campaign to calculate point by point nonlinear light curve fits to light curves obtained during XCOV26. High signal-to-noise light curves $(\mathrm{S} / \mathrm{N} \approx$ 1000) are vital, since we are interested in the nonlinear portion of the data, which is smaller than the linear component. We chose the SOAR $4 \mathrm{~m}$ light curves and the two longest runs from the McDonald $2.1 \mathrm{~m}$ (selected runs are marked with “*” in Table 1). These six runs span 3 days, a timebase long enough to constrain the phases of the closely spaced frequencies, yet short enough to avoid possible implications of amplitude modulation found in our frequency analysis (see Section 3.1).
The dominant frequencies can be considered to be stable over a 3 day timescale. Using model atmosphere tables provided by $\mathrm{D}$. Koester, we calculate the conversion from bolometric to the observed bandpass as described in Montgomery et al. (2010b), assuming the following parameters for this star: $T_{\text {eff }, 0}=$ $11768 \pm 23 \mathrm{~K}$ and $\log g=8.08 \pm 0.008$ in cgs units (Koester et al. 2009).

We began the fitting process by including the frequencies in Table 4 with firm $(l, m)$ identifications. We then included the additional frequencies with strong $(l, m)$ constraints. It is prudent to point out that this fitting process is nonlinear. Adding additional frequencies based on criteria such as amplitude is not necessarily the best procedure. We experimented extensively by computing numerous fits encompassing a wide range of $(l, m)$ identifications for the lower amplitude frequencies. The values of $\tau_{0}$ for all fits range from 99 to $230 \mathrm{~s}$, indicating that $\tau_{0}$ is not strongly dependent on these identifications.

Our investigation does reveal that the temperature parameter $N$ can be highly sensitive to the input $m$ identification for large amplitude modes. Numerous attempts to fit EC14012-1446's light curve while assigning the $1633.907 \mu \mathrm{Hz}$ mode an $(l, m)$ identification of $(1,0)$ as derived from our combination analysis (see Section 4.1) yielded $\tau_{0}=171 \mathrm{~s}, \theta=28^{\circ}$, and $N=39$. While the values of $\tau_{0}$ and $\theta$ are reasonable values based on MLT, the value of $N$ implies a temperature sensitivity that is far below the predictions of MLT (Montgomery et al. 2010b); this is not a physically relevant fit. We also experimented by assigning a spherical index $l=2$ to this mode, resulting in the following fit parameters: $\tau_{0}=228 \mathrm{~s}, N=26$, and $\theta=15^{\circ}$. Again, the value of $N$ obtained remains too low. Ising \& Koester (2001) warn that a perturbation analysis of combination amplitudes (Wu 2001) may have difficulties for photometric variations of high amplitude, and this mode has the largest amplitude in the data set. In addition, the asteroseismology analysis of the $1633 \mu \mathrm{Hz}$ multiplet structure cannot solidly constrain the $m$ value for this mode (see Section 4.2). Following this reasoning, we experimented by assigning the $1633.907 \mu \mathrm{Hz}$ mode an $(l, m)$ 


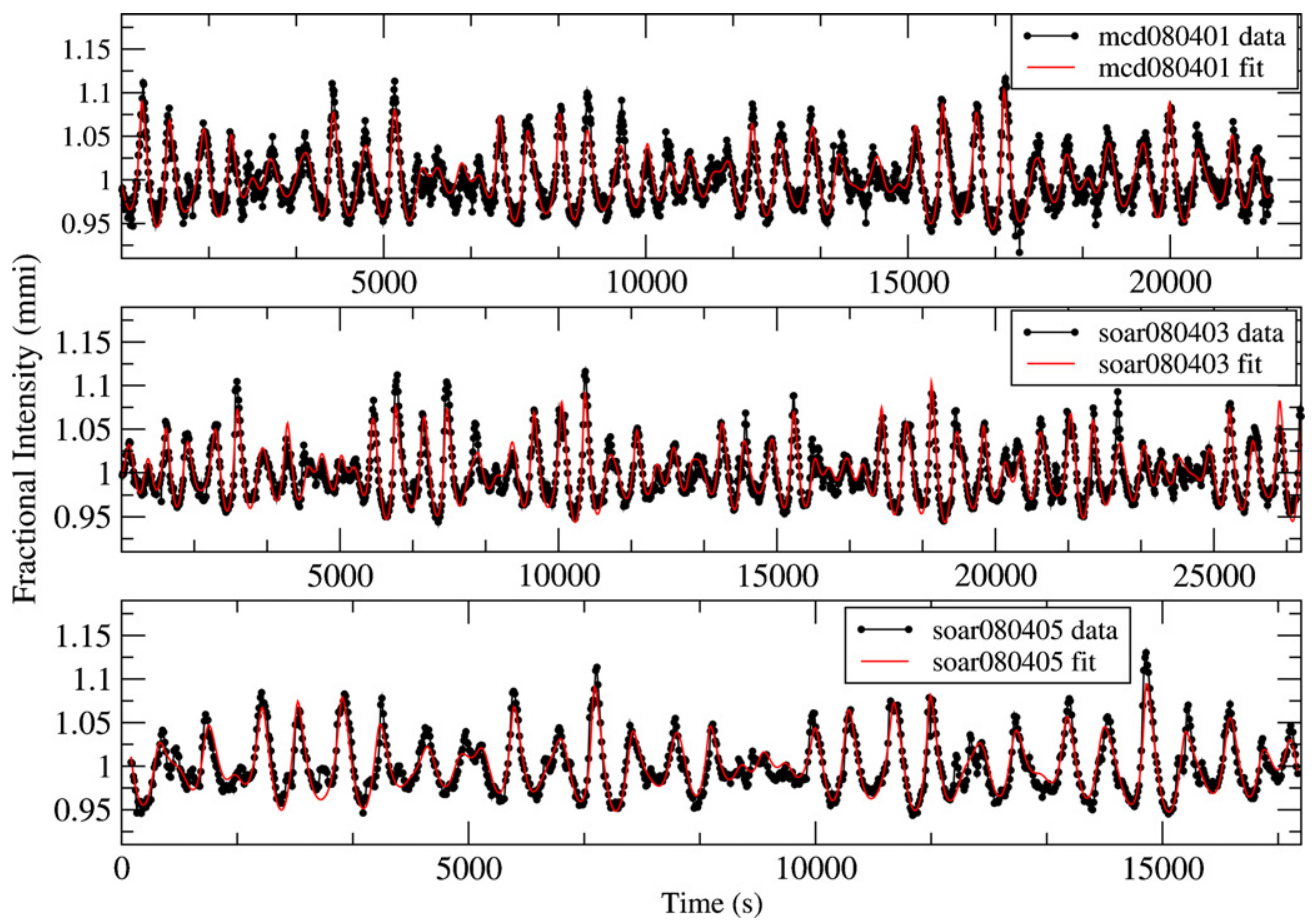

Figure 16. Simultaneous fit of the periods of 19 modes (solid line) to the light curves from mcdo080401, soar080403, and soar080405 (filled circles). Note the change in the $x$-axis for each plot.

(A color version of this figure is available in the online journal.)

value of $(1,1)$. The resulting fit finds $N=85$, in much better agreement with MLT predictions (Wu 2001; Montgomery 2005a).

The final simultaneous nonlinear fit to the six high signalto-noise light curves includes the frequencies and $(l, m)$ identifications given in Table 4. This fit produced the following parameters: $\tau_{0}=99.4 \pm 12 \mathrm{~s}, N=85 \pm 6$, and $\theta=32.9 \pm 3.2$. Figure 16 shows the ability to reproduce the essential features of the light curves.

\section{DISCUSSION}

Convective energy transport in stellar environments is typically modeled using MLT. A particular version by Böhm \& Cassinelli (1971), denoted as ML2, includes reduced horizontal energy loss relative to the formulation of Böhm-Vitense (ML1; 1958), increasing the overall convective efficiency. ML2 has been the standard convection model adopted for stellar atmosphere fits of white dwarfs for the past 20 years, with $\alpha=0.6$ the preferred value for the mixing length (Bergeron et al. 1995). Tremblay et al. (2010) recently re-calibrated the assumed convective efficiency for white dwarf models, using model spectra incorporating an improved treatment of Stark broadening (Tremblay \& Bergeron 2009). Tremblay et al. (2010) fit an improved set of Hubble Space Telescope and International Ultraviolet Explorer ultraviolet (UV) and near UV spectra, varying $\alpha$ until reaching agreement between the optical and UV temperatures. They find the best internal consistency between optical and UV effective temperatures and $\log g$ measurements using ML2 with $\alpha=0.8$. This is a much more efficient version of MLT than found by Bergeron et al. (1995), but is in closer agreement with that required by nonadiabatic models to fit the observed blue edge of the DA instability strip (Fontaine \& Brassard 2008). This convective parameterization is becoming the standard for DA model atmospheres (Freytag et al. 2012; Kilic et al. 2012).

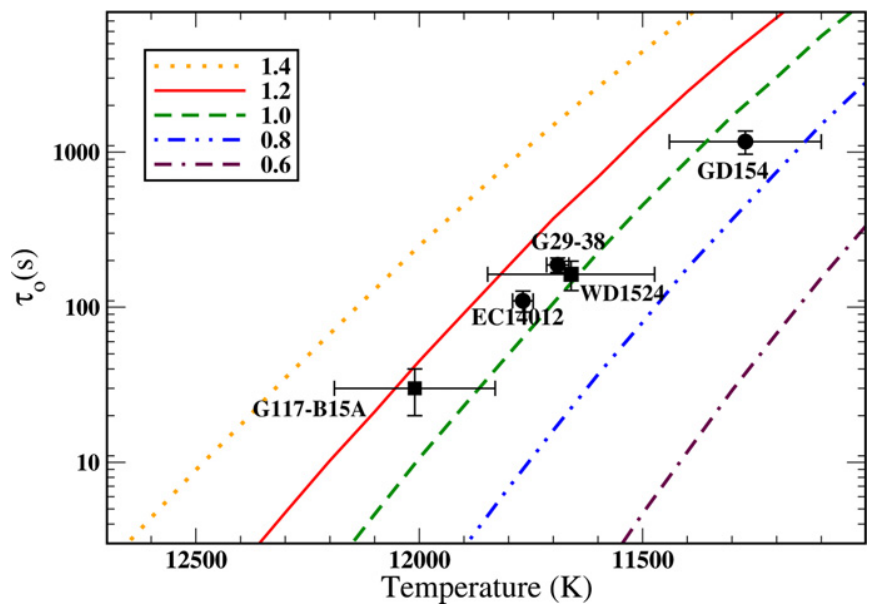

Figure 17. Comparison of EC14012-1446's derived convective parameters with values expected from ML2 convection. The additional labeled points are individual objects taken from Montgomery (2005a), Montgomery et al. (2010a), and Provencal et al. (2011). The curves represent ML2 theoretical calculations of the thermal response time $\tau_{0}$ for various values of the mixing length $\alpha$ $(\log g=8.0)$.

(A color version of this figure is available in the online journal.)

One of our goals is to produce an empirical map of the timeaveraged convective timescale $\tau_{0}$ at the base of the convection zone as a function of $T_{\text {eff }}$ and $\log g$ for a population spanning the DAV instability strip $(\approx 11,100-12,200 \mathrm{~K})$ and compare this with MLT predictions of $\tau_{0}$. As an individual DA white dwarf cools through the instability strip, the base of its surface hydrogen convection zone will deepen, increasing its total mass and the value of $\tau_{0}$. MLT makes specific predictions for the behavior of $\tau_{0}$ given different choices of input parameters. For instance, Figure 17 shows that ML2 with $\alpha=1.0$ predicts $\tau_{0}$ values ranging from $\sim 1 \mathrm{~s}$ at the blue edge of the instability strip to $\sim 6000 \mathrm{~s}$ at the red edge. 
Table 6

Stellar Parameters

\begin{tabular}{|c|c|c|c|c|c|c|c|}
\hline Star & $\begin{array}{l}T_{\text {eff }} \\
(\mathrm{K})\end{array}$ & $\log g$ & $\begin{array}{c}\text { Period } \\
\text { (s) }\end{array}$ & $\begin{array}{l}\tau_{0} \\
(\mathrm{~s})\end{array}$ & $P_{\max }$ & $\theta$ & $N$ \\
\hline G117-B15A & $12010 \pm 180$ & 8.33 & 215.2 & $30 \pm 10$ & $188 \pm 63$ & - & - \\
\hline EC14012-1446 & $11768 \pm 23$ & 8.11 & 612.3 & $99 \pm 12$ & $624 \pm 75$ & $33 \pm 3$ & $85 \pm 6$ \\
\hline G29-38 & $11690 \pm 120$ & 8.11 & 615.2 & $187 \pm 20$ & $1177 \pm 126$ & $66 \pm 3$ & $95 \pm 8$ \\
\hline WDJ1524-0030 & $11660 \pm 180$ & 8.06 & 697.3 & $163 \pm 35$ & $1024 \pm 220$ & $58 \pm 10$ & $95 \pm 15$ \\
\hline GD154 & $11270 \pm 170$ & 7.95 & 1185.9 & $1169 \pm 200$ & $7345 \pm 1257$ & $10 \pm 10$ & $127 \pm 25$ \\
\hline
\end{tabular}

Notes. $T_{\text {eff }}$ measurements are from Koester et al. (2009), Koester \& Holberg (2001), and Koester \& Allard (2000). Given pulsation periods are for the largest amplitude frequency. Entries marked with "_" did not have unique values determined by the fits.

In Figure 17 we also plot current determinations of $\tau_{0}$ versus $T_{\text {eff }}$ for five DAVs, including our solution for EC14012-1446; the derived stellar parameters are listed in Table 6 . To ensure a uniform treatment of all the stars in our sample, we have used $T_{\text {eff }}$ determinations that do not include the recent updates to the line profile calculations by Tremblay \& Bergeron (2009); rather, the plotted effective temperatures and horizontal error bars are based on earlier spectroscopic fits employing ML2 (Koester et al. 2009; Koester \& Holberg 2001; Koester \& Allard 2000). Overlaid on this figure are the $\tau_{0}$ predictions of ML2 convection for various values of the mixing length parameter $\alpha$. Our current results do indicate an increase in $\tau_{0}$ (and hence an increase in depth and mass of the convection zone) with decreasing temperature and are marginally consistent with $\alpha=1.0$. Decreasing the $T_{\text {eff }}$ error bars, either through higher signal-to-noise spectra and/or new model atmosphere fits, will provide more precise constraints on convection in these stars.

Finally, we point out that convective light curve fitting extracts the value of $\tau_{0}$ at the base of the convection zone. There is no reason why the same value of $\alpha$ should describe both the photosphere and the deeper convective layers, so our results do not necessarily have to agree with the results of Tremblay et al. (2010) or Bergeron et al. (1995). For instance, Ludwig et al. (1994) use sophisticated two-dimensional hydrodynamic simulations to show that, while MLT is a reasonable approximation to predict the rough photospheric temperature structure of DA white dwarfs, the deeper layers have a higher convective efficiency than predicted by MLT.

Our treatment of the nonlinearities in white dwarf light curves is based on the larger picture of how a surface convection zone leads to driving in these stars. In particular, Brickhill (1991) and Goldreich \& Wu (1999) demonstrate that excitation of $g$-mode pulsations should occur when the convective driving exceeds the radiative damping, and that this condition is given by $\omega \tau_{0} \gtrsim 1$. In terms of period, this relation states that $g$-modes should be driven when $P \lesssim P_{\max }$, where $P_{\max } \equiv 2 \pi \tau_{0}$ and $P$ is the period of a given mode. In Figure 18 we compare the dominant oscillation period in each of the DAVs we have fit with their theoretical value of $P_{\max }$, as calculated from each star's value of $\tau_{0}$. The agreement is good for $\tau_{0} \lesssim 100 \mathrm{~s}$, but the values diverge for larger values of $\tau_{0}$. Given that $\tau_{0}$ is related to a star's $T_{\text {eff }}$ (see Figure 17), this says that the agreement is good from the blue edge to near the middle of the instability strip, but that from this point to the red edge another effect is operating that prevents the dominant mode periods from increasing as rapidly as $P_{\max }$.

It is natural to assume that the cause of this behavior is related to whatever unknown phenomenon produces the red edge of the instability strip. Wu \& Goldreich (2001) point out that a deepening convection zone attenuates the flux variations entering at its base, so that for deep enough convection zones the surface amplitudes will be below detection limits. However,

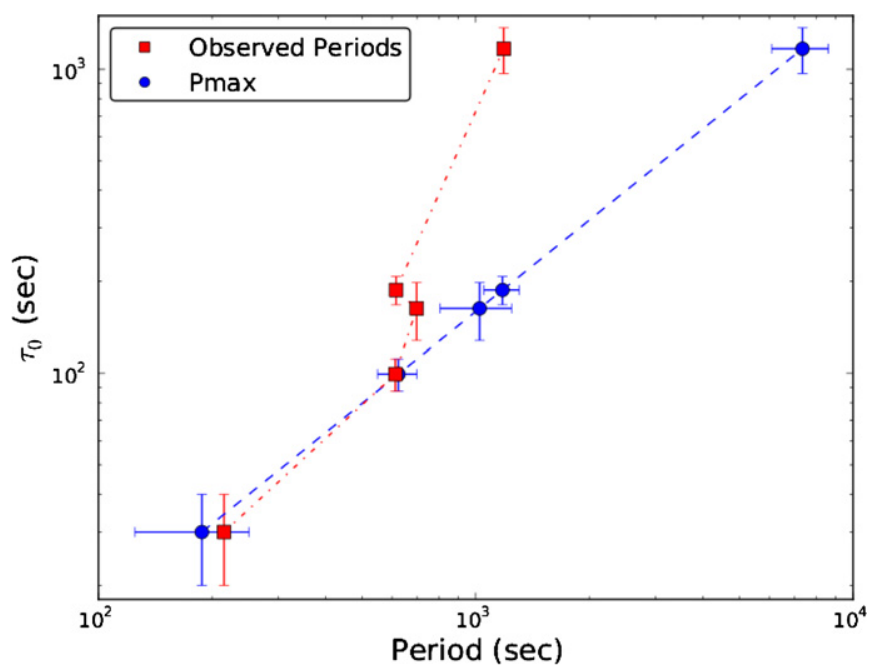

Figure 18. Comparison of the dominant observed pulsation period in a star (dot-dashed curve, red squares) with the theoretical value of $P_{\max }$ (dashed line, blue circles) for each DAV star with a measured value of $\tau_{0}$. Cooler stars are to the left, hotter stars to the right. While simple theory predicts that these curves should be very similar, we find a significant departure for cooler stars.

(A color version of this figure is available in the online journal.)

this is an amplitude effect and does not predict that the dominant periods should be different from $P_{\max }$. Brickhill (1991), Goldreich \& Wu (1999), and Wu \& Goldreich (2001) also discuss the importance of turbulent damping in a narrow shear layer at the base of the convection zone; damping from this region may be more significant for cooler stars. Another possibility is that the maximum period for which complete surface reflection of $g$-modes can occur is much lower than expected; typical estimates place this number on the order of $\sim 5000 \mathrm{~s}$ for $l=1$ modes and $\sim 3000 \mathrm{~s}$ for $l=2$ modes (Hansen et al. 1985), although it is possible that improved treatment of the surface layers could alter these estimates. At any rate, this phenomenon is likely related to the deepening of the convection zone toward the red edge; we are exploring other phenomena that could lead to this behavior.

\section{CONCLUSIONS}

Until the advent of convective light curve fitting, our Sun was the only star with empirical constraints on its convection zone depth. Determinations of the time-averaged convective timescale $\tau_{0}$ and the temperature sensitivity parameter $N$ can now potentially be obtained for any white dwarf pulsator of moderate amplitude (Montgomery et al. 2010b); approximately two-thirds of all white dwarf pulsators show significant nonlinearities in their light curves. Our ultimate goal is to map $\tau_{0}$ as a function of $T_{\mathrm{eff}, 0}$ and $\log g$ for a population spanning the 
instability strips of both the DAV and DBV white dwarfs. Such a map will provide important empirical constraints on convection for white dwarfs and eventually other types of pulsating stars.

We have taken the first steps in this direction with our investigation of EC14012-1446. XCOV26 produced $308.3 \mathrm{hr}$ of data, and our analysis has identified 19 independent frequencies distributed in 14 multiplets. Combined with archival observations, we have identified a series of $l=1$ modes with an average period spacing of 41 s. EC14012-1446 is now one of the few DAVs with over a dozen identified modes in its pulsation spectrum. The large number of modes means that asteroseismology can be used to provide constraints on its interior structure.

Future goals focus on the determination of $\tau_{0}$ and $N$ for additional white dwarf pulsators. An increased sample size will improve our empirical map of $\tau_{0}$ as a function of $T_{\text {eff }}$ and also allow us to further explore the observed behavior of $P$ and $P_{\max }$ as a function of $\tau_{0}$. Our goals also include the reduction of errors associated with spectroscopic temperatures. Convective light curve fitting demands uniform treatment of convection between the spectroscopic temperatures we choose and the temperatures we use to calculate the light curve fits. Since WD1524-0030 was not included in the new temperatures of Gianninas et al. (2011) that incorporate recent updates to line profile calculations, we use a consistent set of older published temperatures for the objects presented in this work. Incorporating the new temperatures will be a first step toward reducing the spectroscopic errors. We must recalculate our existing nonlinear light curve fits using these updated $T_{\text {eff }}$ and $\log g$ determinations. On average, the temperatures of Gianninas et al. (2011) increase over older published values by $\approx 500 \mathrm{~K}$. Preliminary work indicates that the empirical value of $\tau_{0}$ will not change significantly with such an increase. However, the theoretical MLT predictions for $\tau_{0}$ will change; larger values of $\alpha$ will be needed to keep $\tau_{0}$ and the convection zone depth the same for these higher values of $T_{\text {eff }}$.

The Delaware Asteroseismic Research Association (DARC) is grateful for the support of the Crystal Trust Foundation and Mt. Cuba Observatory. DARC also acknowledges the support of the University of Delaware, through their participation in the SMARTS consortium. M.H.M. gratefully acknowledges the support of the NSF under grant AST-0909107 and the Norman Hackerman Advanced Research Program under grant 003658-0252-2009. S.L.K. acknowledges partial support by the KASI (Korea Astronomy and Space Science Institute) grant 2012-1-410-02. This paper uses observations made at the South African Astronomical Observatory (SAAO). This work is further supported by the Austrian Fonds zur Förderung der wissenschaftlichen Forschung under grant P18339-N08. We thank the various Telescope Allocation Committees for the awards of telescope time.

Facilities: MCAO:0.6m, Struve, KPNO:2.1m, UH:0.6m, BOAO:1.8m, Lulin:1.8m, Beijing:2.16m, Maidanek:1.0m, Peak Terskol, SAAO:1.0m ( ), SALT, SOAR, CTIO:0.9m, LCOGT, BOAO: $1.8 \mathrm{~m}$.

\section{REFERENCES}

Berger, L., Koester, D., Napiwotzki, R., Reid, I. N., \& Zuckerman, B. 2005, A\&A, 444, 565

Bergeron, P., Wesemael, F., Lamontagne, R., et al. 1995, ApJ, 449, 258

Biermann, L. 1932, Z. Astrophys., 5, 117

Bischoff-Kim, A., \& Metcalfe, T. 2012, in ASP Conf. Ser., Progress in Solar and Stellar Physics with Asterseismology, ed. H. Shibahashi, in press
Böhm, K. H., \& Cassinelli, J. 1971, A\&A, 12, 21

Böhm-Vitense, E. 1958, Z. Astrophys., 46, 108

Brassard, P., Fontaine, G., \& Wesemael, F. 1995, ApJS, 96, 545

Brickhill, A. J. 1991, MNRAS, 251, 673

Brickhill, A. J. 1992, MNRAS, 259, 519

Charbonneau, P. 1995, ApJS, 101, 309

Córsico, A. H., Althaus, L. G., Benvenuto, O. G., \& Serenelli, A. M. 2002, A\&A, 387,531

Córsico, A. H., Althaus, L. G., Kawaler, S. D., et al. 2011, MNRAS, 418, 2519

Dolez, N., Vauclair, G., Kleinman, S. J., et al. 2006, A\&A, 446, 237

Eisenstein, D. J., Liebert, J., Koester, D., et al. 2006, AJ, 132, 676

Fontaine, G., \& Brassard, P. 2008, PASP, 120, 1043

Freytag, B., Steffen, M., Ludwig, H.-G., et al. 2012, J. Comput. Phys., 231, 919

Gianninas, A., Bergeron, P., \& Ruiz, M. T. 2011, ApJ, 743, 138

Goldreich, P., \& Wu, Y. 1999, ApJ, 511, 904

Handler, G., Romero-Colmenero, E., \& Montgomery, M. H. 2002, MNRAS, 335,399

Handler, G., Romero-Colmenero, E., Provencal, J. L., et al. 2008, MNRAS, 388,1444

Hansen, C. J., Winget, D. E., \& Kawaler, S. D. 1985, ApJ, 297, 544

Harris, H. C., Munn, J. A., Kilic, M., et al. 2006, AJ, 131, 571

Ising, J., \& Koester, D. 2001, A\&A, 374, 116

Kanaan, A., Kepler, S. O., \& Winget, D. E. 2002, A\&A, 398, 896

Kawaler, S. E. 1988, in IAU Symp. 123, Advances in Helio- and Asteroseismology, ed. J. Christensen-Dalsgaard \& S. Frandsen (Dordrecht: Reidel), 329

Kepler, S. O., Nather, R. E., Winget, D. E., et al. 2003, A\&A, 401, 639

Kilic, M., Patterson, A. J., Barber, S., Leggett, S. K., \& Dufour, P. 2012, MNRAS, 419, L59

Koester, D., \& Allard, N. 2000, Balt. Astron., 9, 119

Koester, D., \& Holberg, J. 2001, in ASP Conf. Ser. 226, 12th European Conference on White Dwarfs, ed. J. L. Provencal, H. L. Shipman, J. MacDonald, \& S. Goodchild (San Francisco, CA: ASP), 299

Koester, D., Voss, B., Napiwotzki, R., et al. 2009, A\&A, 505, 441

Lenz, P., \& Breger, M. 2005, Commun. Asteroseismol., 146, 53

Ludwig, H., Jordan, S., \& Steffen, M. 1994, A\&A, 284, 105

Metcalfe, T. S., Nather, R. E., \& Winget, D. E. 2000, ApJ, 545, 974

Montgomery, M. H. 2005a, ApJ, 633, 1142

Montgomery, M. H. 2005b, in ASP Conf. Ser. 334, 14th European Workshop on White Dwarfs, ed. D. Koester \& S. Moehler (San Francisco, CA: ASP), 553

Montgomery, M. H. 2005c, in ASP Conf. Ser. 334, 14th European Workshop on White Dwarfs, ed. D. Koester \& S. Moehler (San Francisco, CA: ASP), 483

Montgomery, M. H. 2007, in ASP Conf. Ser. 372, 15th European Workshop on White Dwarfs, ed. R. Napiwotzki \& M. R. Burleigh (San Francisco, CA: ASP), 635

Montgomery, M. H. 2009, in AIP Conf. Proc. 1170, Stellar Pulsation: Challenges for Theory and Observation, ed. J. A. Guzik \& P. A. Bradley (Melville, NY: AIP), 605

Montgomery, M. H., Hermes, J. J., \& Winget, D. E. 2010a, in AIP Conf. Proc. 1273, 17th European White Dwarf Workshop, ed. K. Werner (Melville, NY: AIP), 512

Montgomery, M. H., Provencal, J. L., Kanaan, A., et al. 2010b, ApJ, 716, 84

Provencal, J. L., Montgomery, M. H., Kanaan, A., et al. 2009, ApJ, 693, 564

Provencal, J. L., Montgomery, M. H., Mullaly, S., Dalessio, J., \& Shipman, H. 2011, BAAS, 43, 21734105

Ruiz, M. T., \& Bergeron, P. 2001, ApJ, 558, 761

Stobie, R. S., O’Donoghue, D. O., Ashley, R., et al. 1995, MNRAS, 272, L21

Tassoul, M., Fontaine, G., \& Winget, D. E. 1990, ApJS, 72, 335

Thompson, S. E., \& Mullally, F. 2009, J. Phys.: Conf. Ser., 172, 012081

Tremblay, P. E., \& Bergeron, P. 2009, in AIP Conf. Proc. 1171, Recent Directions in Astrophysical Quantitative Spectroscopy and Radiation Hydrodynamics (Melville, NY: AIP), 101

Tremblay, P.-E., Bergeron, P., Kalirai, J. S., \& Gianninas, A. 2010, ApJ, 712, 1345

Unno, W., Osaki, Y., Ando, H., Saio, H., \& Shibahashi, H. 1989, Nonradial Oscillations of Stars (Tokyo: Univ. Tokyo Press)

Winget, D. E., Hansen, C. J., Liebert, J., et al. 1987, ApJ, 315, L77

Winget, D. E., Nather, R. E., Clemens, J. C., et al. 1991, ApJ, 378, 326

Winget, D. E., Nather, R. E., Clemens, J. C., et al. 1994, ApJ, 430, 839

Wu, Y. 2001, MNRAS, 323, 248

Wu, Y., \& Goldreich, P. 2001, ApJ, 546, 469

Yeates, C. M., Clemens, J. C., Thompson, S. E., \& Mullally, F. 2005, ApJ, 635, 1239 